\title{
A VLA SURVEY FOR FAINT COMPACT RADIO SOURCES IN THE ORION NEBULA CLUSTER
}

\author{
Patrick D. Sheehan ${ }^{1}$, Josh A. Eisner ${ }^{1}$, Rita K. ManN ${ }^{2}$, and Jonathan P. Williams ${ }^{3}$ \\ ${ }^{1}$ Steward Observatory, University of Arizona, 933 N. Cherry Avenue, Tucson, AZ 85721, USA; psheehan@email.arizona.edu \\ 2 National Research Council Canada, 5071 West Saanich Road, Victoria, BC, V9E 2E7, Canada \\ ${ }^{3}$ Institute for Astronomy, University of Hawaii at Manoa, Honolulu, HI 96822, USA \\ Received 2016 February 22; revised 2016 August 7; accepted 2016 August 26; published 2016 November 4
}

\begin{abstract}
We present Karl G. Jansky Very Large Array 1.3, 3.6, and $6 \mathrm{~cm}$ continuum maps of compact radio sources in the Orion Nebular Cluster (ONC). We mosaicked $34 \operatorname{arcmin}^{2}$ at $1.3 \mathrm{~cm}, 70 \operatorname{arcmin}^{2}$ at $3.6 \mathrm{~cm}$ and $109 \operatorname{arcmin}^{2}$ at $6 \mathrm{~cm}$, containing 778 near-infrared detected young stellar objects and 190 Hubble Space Telescope-identified proplyds (with significant overlap between those characterizations). We detected radio emission from 175 compact radio sources in the ONC, including 26 sources that were detected for the first time at these wavelengths. For each detected source, we fitted a simple free-free and dust emission model to characterize the radio emission. We extrapolate the free-free emission spectrum model for each source to ALMA bands to illustrate how these measurements could be used to correctly measure protoplanetary disk dust masses from submillimeter flux measurements. Finally, we compare the fluxes measured in this survey with previously measured fluxes for our targets, as well as four separate epochs of $1.3 \mathrm{~cm}$ data, to search for and quantify the variability of our sources.
\end{abstract}

Key words: protoplanetary disks - radio continuum: stars - stars: formation

Supporting material: extended figures, machine-readable tables

\section{INTRODUCTION}

The Orion Nebular Cluster (ONC) presents an excellent example of star formation in a richly clustered environment, typical of star formation in our galaxy. Near-infrared surveys of the ONC find $>700$ young stellar objects (YSOs), most of which are likely to harbor protoplanetary disks (Hillenbrand \& Carpenter 2000). Hubble Space Telescope (HST) images of the ONC also reveal ionized disks and dusty disks silhouetted against the backdrop of nebular emission (e.g., O'Dell \& Wen 1994; Bally et al. 1998a; Smith et al. 2005; Ricci et al. 2008).

The O6 star $\theta^{1}$ Ori $\mathrm{C}$, located in the central Trapezium Cluster, produces intense UV radiation that photoevaporates many of the nearby protoplanetary disks. The hot gas ionized by this intense radiation expands freely and flows away at the local sound speed into lower pressure regions (e.g., Henney \& Arthur 1998). The ionized winds from the protoplanetary disks emit strong free-free emission at radio wavelengths (e.g., Churchwell et al. 1987; Garay et al. 1987).

Compact radio sources have long been known in the ONC (e.g., Moran et al. 1982; Churchwell et al. 1987; Garay et al. 1987; Felli et al. 1993a; Zapata et al. 2004a, 2004b). They were first identified as free-free emission by Garay et al. (1987), and suggested to be the ionized material evaporated from protostellar disks by Churchwell et al. (1987). Observations of the ONC with the HST firmly established these compact structures as externally ionized protoplanetary disks (e.g., O'Dell et al. 1993).

Measurements of the masses of protoplanetary disks are crucial for understanding evolution, as well as the potential for planet formation. Disk mass measurements are typically made by observing dust continuum emission at long wavelengths, where the emission is optically thin and probes the entirety of the disk (e.g., Beckwith et al. 1990). Toward this end, a host of millimeter interferometric surveys of the ONC have previously been carried out (e.g., Mundy et al. 1995; Bally et al. 1998b;
Williams et al. 2005; Eisner \& Carpenter 2006; Eisner et al. 2008; Mann \& Williams 2009, 2010; Mann et al. 2014).

These surveys are complicated by potential contamination of the millimeter dust continuum emission by free-free emission from ionized disk winds. Disk mass measurements are facilitated at shorter wavelengths, of $1.3 \mathrm{~mm}$ or $870 \mu \mathrm{m}$, where the ratio of dust emission to free-free emission is expected to be more favorable. Even here, however, free-free emission can contribute significantly to the observed brightness of the sources (e.g., Eisner et al. 2008; Mann \& Williams 2009, 2010; Mann et al. 2014).

Observations at longer radio wavelengths can help to constrain the free-free contribution at shorter wavelengths. Free-free emission has a flat spectrum $\left(F_{\nu} \propto \nu^{-0.1}\right)$ when optically thin, which is expected to be true at millimeter and centimeter wavelengths (e.g., Eisner et al. 2008; Mann \& Williams 2009, 2010; Mann et al. 2014). Optically thick freefree emission can span a range of spectral indices, but the emission usually only becomes optically thick at wavelengths longer than $\sim 10 \mathrm{~cm}$ (e.g., Eisner et al. 2008). Dust emission, however, has a steep spectral index $\left(F_{\nu} \propto \nu^{2+\beta}, \beta=0-2\right)$, which falls off rapidly at longer wavelengths. Free-free emission can therefore be constrained at longer radio wavelengths where the contribution from dust emission to the flux is small. Radio fluxes may also in some cases be affected by magnetospheric flaring from young stars, exhibiting gyrosynchrotron emission with a steep negative spectral index when optically thin $\left(F_{\nu} \propto \nu^{-0.7}\right.$; e.g., Feigelson \& Montmerle 1999; Rivilla et al. 2015), or a steep positive spectral index when optically thick at lower frequencies $\left(F_{\nu} \propto \nu^{2.5}\right)$.

Previous studies have used the Very Large Array (VLA) to search for compact radio sources in the ONC (e.g., Felli et al. 1993a; Zapata et al. 2004a), and fluxes produced by those studies have been used to correct for free-free contamination in disk mass studies (e.g., Eisner et al. 2008; Mann \& Williams 2010; Mann et al. 2014). The expanded capabilities of the VLA correlator (Perley et al. 2009), now enable surveys of much 
Table 1

Log of VLA Observations

\begin{tabular}{|c|c|c|c|c|c|c|c|c|c|c|}
\hline Band & Configuration & Date & $\begin{array}{l}\text { Int. Time } \\
\text { (minutes) }\end{array}$ & $\begin{array}{l}\mathrm{rms} \\
(\mu \mathrm{Jy})\end{array}$ & $\begin{array}{l}\text { Peak rms } \\
(\mu \mathrm{Jy})\end{array}$ & Beam & No. Beams & $\begin{array}{c}\text { Total } \\
\text { Detections }\end{array}$ & $\begin{array}{c}>6 \sigma \\
\text { Detections }\end{array}$ & $\begin{array}{c}>4.5 \sigma \\
\text { Detections }\end{array}$ \\
\hline $1.3 \mathrm{~cm}$ & B & 2013 Nov 10 & 62 & 33 & $\sim 93$ & $0 " .33 \times 0$ ". 21 & $1.5 \times 10^{6}$ & 79 & 57 & 22 \\
\hline $6 \mathrm{~cm}$ & A & 2014 Mar 3 & 7 & 37 & $\sim 150$ & $0 " .40 \times 0 . ! 28$ & $3.1 \times 10^{6}$ & 108 & 87 & 21 \\
\hline $3.6 \mathrm{~cm}$ & A & 2014 Mar 3 & 9.5 & 30 & $\sim 70$ & $0 ! .24 \times 0$ "! 18 & $5.1 \times 10^{6}$ & 98 & 80 & 18 \\
\hline $1.3 \mathrm{~cm}$ & A & 2014 Mar 3 & 49 & 25 & $\sim 50$ & $0 . " 09 \times 0 . " 08$ & $14.9 \times 10^{6}$ & 70 & 54 & 16 \\
\hline $1.3 \mathrm{~cm}$ & A & 2014 Mar 7 & 49 & 26 & $\sim 100$ & $0 . " 08 \times 0 . " 08$ & $15.7 \times 10^{6}$ & 73 & 56 & 17 \\
\hline $1.3 \mathrm{~cm}$ & A & 2014 May 3 & 36.5 & 22 & $\sim 85$ & $0 ! .10 \times 0 . ! 07$ & $14.6 \times 10^{6}$ & 89 & 67 & 22 \\
\hline $1.3 \mathrm{~cm}$ & A \& B combined & $\ldots$ & $\ldots$ & 12 & $\sim 50$ & $0 ! 09 \times 0 . " 09$ & $12.4 \times 10^{6}$ & 126 & 98 & 28 \\
\hline
\end{tabular}

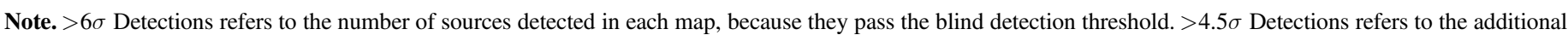
sources detected in a catalog-driven search, and Total Detections is the sum of those numbers.

higher sensitivity than were previously possible. More recent surveys have taken advantage of this increase in sensitivity to map star-forming regions, including the ONC at 4.5 and 7.5 GHz (Dzib et al. 2013; Kounkel et al. 2014; Forbrich et al. 2016). This enhanced sensitivity is well matched to the deeper observations now enabled with ALMA.

Here, we present new high-resolution Karl G. Jansky Very Large Array (henceforth JVLA to avoid confusion with previous surveys using the original VLA) maps of the ONC at $1.3,3.6$, and $6 \mathrm{~cm}$ to study the free-free emission from ONC cluster members. In Section 2 we describe our observations and maps of the ONC. In Section 3 we detail our methodology for searching for compact radio sources, as well as our model for characterizing the free-free emission. In Section 4 we compare our results to previous catalogs of compact radio sources in the ONC, discuss the nature of the sources we detect, and show that our measurements are crucial for accurately measuring disk masses of protoplanetary disks from both current and future submillimeter surveys.

\section{OBSERVATIONS AND DATA REDUCTION}

We imaged the $\mathrm{ONC}$ in $1.3,3.6$, and $6 \mathrm{~cm}$ wavelength continuum emission with the JVLA between 2013 November and 2014 May. The 3.6 and $6 \mathrm{~cm}$ maps were observed using the "A" configuration (baselines ranging from $680 \mathrm{~m}$ to $36 \mathrm{~km}$ ), and the $1.3 \mathrm{~cm}$ data were taken in three epochs with the "A" configuration and one epoch with the "B" configuration (baselines ranging from $210 \mathrm{~m}$ to $11 \mathrm{~km}$ ). Details of the observations and maps are provided in Table 1.

The 3.6 and $6 \mathrm{~cm}$ data were taken simultaneously in 32 $128 \mathrm{MHz}$ bands, split evenly between 3.6 and $6 \mathrm{~cm}$. Each band contained $642 \mathrm{MHz}$ channels, and the bands were arranged continuously from $4.488-6.512 \mathrm{GHz}$ at $6 \mathrm{~cm}$ and from $8.116-10.012 \mathrm{GHz}$ at $3.6 \mathrm{~cm}$, for a total of $2 \mathrm{GHz}$ of continuum bandwidth each.

The field of view of the JVLA antenna primary beam at $6 \mathrm{~cm}$, FWHM of $9^{\prime}$, encompasses all 778 YSOs from Hillenbrand \& Carpenter (2000), and all of the 196 HSTdetected proplyds (Ricci et al. 2008). 141 of the 196 HSTdetected proplyds are also detected as sources in Hillenbrand \& Carpenter (2000). We, therefore, use a single pointing to image the field at $6 \mathrm{~cm}$. At $3.6 \mathrm{~cm}$ the field of view is $5^{\prime}$, so we imaged the field with two pointings that encompassed 778 YSOs and 187 HST-detected proplyds.

For a rectangular mosaic, the Nyquist sampling theorem suggests that a pointing spacing of $\mathrm{FWHM} / 2$ or better is needed (e.g., Cornwell 1988), but since we are interested in compact sources, Nyquist sampling is not crucial (e.g., Eisner et al. 2008). At $3.6 \mathrm{~cm}$, the FWHM/2 is between $2 ! 1$ and $2 ! 6$ across the band. The two $3.6 \mathrm{~cm}$ pointings are separated by $2 ! 4$, so that the map is sub-Nyquist sampled at the low-frequency end of the band, but not at the high-frequency end.

The $1.3 \mathrm{~cm}$ data were taken in $64128 \mathrm{MHz}$ bands arranged from 17.976-26.024 GHz. Each band was composed of 64 $2 \mathrm{MHz}$ channels, for a total of $8 \mathrm{GHz}$ of bandwidth. Most of the data from 17.976-22.024 GHz, however, are affected by significant RFI, so we exclude that data from our analysis. The $1.3 \mathrm{~cm}$ data, therefore, have an effective bandwidth of $4 \mathrm{GHz}$.

A field of view containing 778 YSOs and 193 HST-detected proplyds was mosaicked using seven pointings. A twodimensional map is Nyquist sampled if the pointing spacing is $\mathrm{FWHM} / \sqrt{3}$ or better, but since we are interested here in compact sources, Nyquist sampling is, again, not crucial. At $1.3 \mathrm{~cm} \mathrm{FWHM} / \sqrt{3}$ is between 1.2 and $1 ! 4$ across the band. The mosaic spacings range between $1^{\prime}$ and $2^{\prime}$, so that the map is largely not Nyquist sampled. We show the field of view of our observations for each band in Figure 1.

The data were calibrated and imaged using the CASA software package. Antenna-based complex gains were calculated using periodic observations of the quasar J0541-0541. Bandpass solutions for each antenna were calculated from observations of the quasar J0319+4130, and the overall flux density scale was calculated using models included in CASA for 3C48.

We produced maps of the $\mathrm{ONC}$ at each frequency by Fourier transforming the complex visibilities, using the mosaicking modes for the 1.3 and $3.6 \mathrm{~cm}$ maps. We weighted the data with a robust parameter of 0 , which provided a good balance between the high sensitivity of normal weighting and the high spatial resolution of uniform weighting. We also used the multi-frequency synthesis option with $\mathrm{n}$ terms $=1$ for no source frequency dependence (Rau \&; Cornwell 2011). Our goal is to search for compact structures in the Orion Nebula, and so we removed baselines shorter than $100 \mathrm{k} \lambda$ from our data before inverting the visibilities. The spatial scales eliminated by this cut correspond to structures greater than $2^{\prime \prime}$, meaning that large-scale structure from the Orion Nebula has been resolved out of our maps. For these observations, our reference frequencies are $5.5 \mathrm{GHz}$ for the $6 \mathrm{~cm}$ map, $9 \mathrm{GHz}$ for the $3.6 \mathrm{~cm}$ map, and $22.5 \mathrm{GHz}$ for the $1.3 \mathrm{~cm}$ map. We image the $6 \mathrm{~cm}$ data out to the $20 \%$ gain contour at $5.5 \mathrm{GHz}$, and the smaller $3.6 \mathrm{~cm}$ and $1.3 \mathrm{~cm}$ maps out to the $10 \%$ gain contour at 

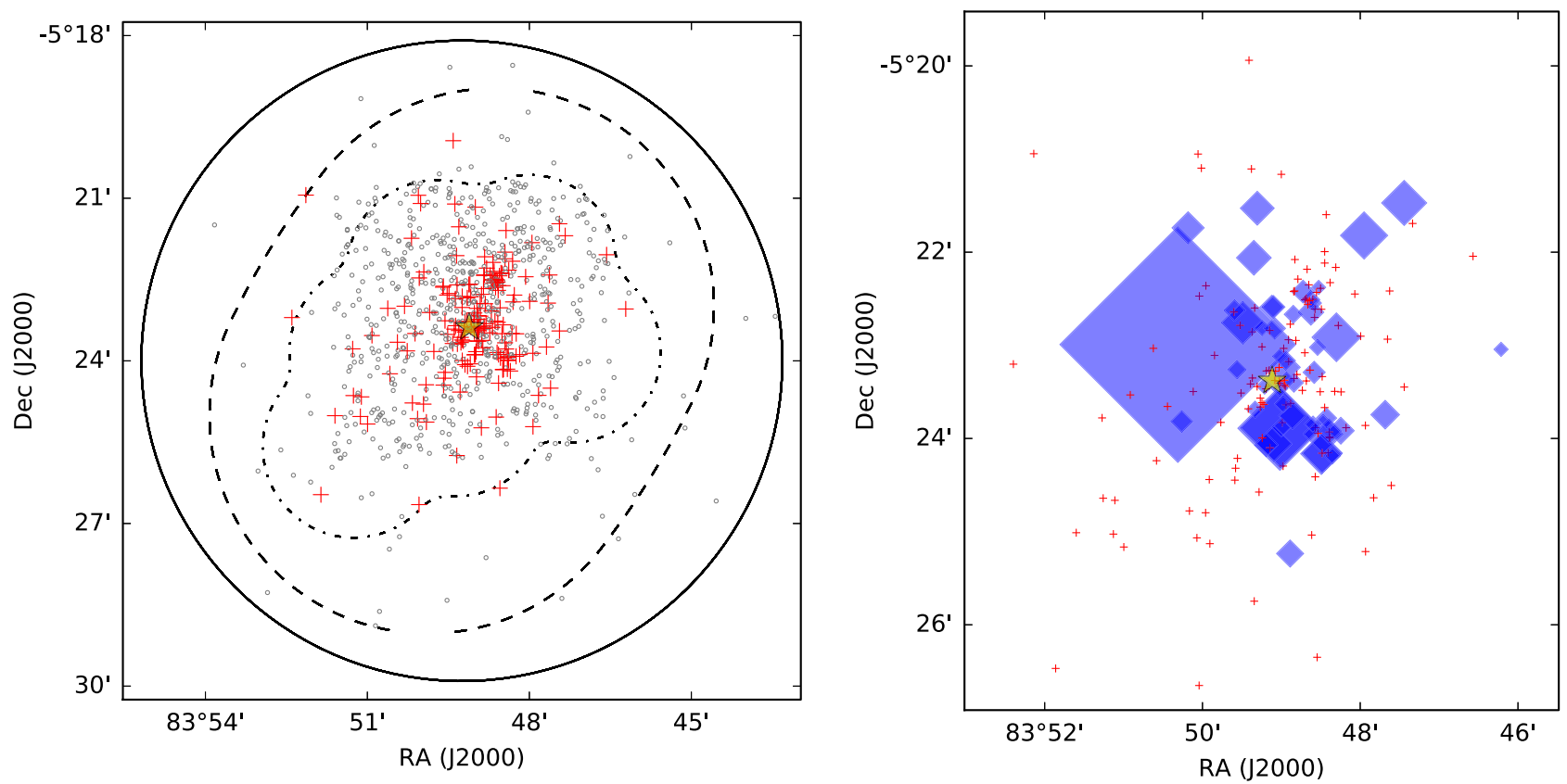

Figure 1. The fields we image, out to the $20 \%$ gain contour at $6 \mathrm{~cm}$ (solid) and the $10 \%$ gain contour at $3.6 \mathrm{~cm}$ (dashed) and $1.3 \mathrm{~cm}$ (dash-dotted) observations, with a yellow star representing the location of $\theta^{1}$ Ori C. On the left, we show all of the sources we detected in at least one of our bands with red plusses and the known sources surveyed, but not detected, with gray circles. On the right, we show sources found to be variable with blue rectangles whose size is proportional to how variable the source is. The largest symbols represent a variability amplitude of $900 \%$, while the smallest represent an amplitude of $20 \%$. The detected sources, which are not variable, are shown again with red plusses.

9 and $22 \mathrm{GHz}$, respectively. ${ }^{4}$ We imaged each $1.3 \mathrm{~cm}$ epoch separately to study the variability of the bright sources, and together to increase our sensitivity to look for faint sources in the map.

We CLEANed the images using the Clark algorithm (Clark 1980). Sources above $10 \sigma$ were initially identified for CLEANing by visual inspection. The maps were CLEANed down to the rms, as measured in source-free regions of the maps, listed in Table 1. Post-source detection, we could reCLEAN the image using the new detections. However, the sidelobes of these sources are low enough to be below the noise level, and the improvement by CLEANing them is minimal and the computational requirements are significant.

We used a single iteration of self-calibration on the $6 \mathrm{~cm}$ data, correcting for just the phases of our data from a model produced by an initial CLEANing of the data. This improved the rms ( $\sim 50 \mu \mathrm{Jy}$ to $\sim 40 \mu \mathrm{Jy})$ in crowded regions of the map or near bright sources with significant beam artifacts.

We self-calibrated the data using a model produced from both fields simultaneously. We find that self-calibrating the fields separately and then imaging them jointly produced ringing in the image that was removed by self-calibrating the data together. We used two iterations of self-calibration, first solving for the phases from our initial model, and then solving for the amplitudes and any residual phase errors in a second iteration. We apply amplitude self-calibration, because it helps to remove residual artifacts around bright sources in our map. It does not change the flux in our maps markedly. This improved the rms from $\sim 80 \mu \mathrm{Jy}$ near bright sources with significant beam artifacts to $\sim 45 \mu \mathrm{Jy}$.

\footnotetext{
4 These correspond to the $33 \%$ and $10 \%$ gain contours for the low- and highfrequency $6 \mathrm{~cm}$ band edges, respectively, the $16 \%$ and $6 \%$ gain contours for the $3.6 \mathrm{~cm}$ band edges, and the $14 \%$ and $6 \%$ gain contours for the $1.3 \mathrm{~cm}$ band edges.
}

We self-calibrated fields including the brightest sources together, which is necessary to remove ringing like in the $3.6 \mathrm{~cm}$ maps, using a single iteration of self-calibration to correct phase errors in the data. The self-calibration improved the rms by as much as a factor of 3 near bright sources with significant beam artifacts (e.g., $\sim 50 \mu \mathrm{Jy}$ to $\sim 20 \mu \mathrm{Jy}$ for the combined $1.3 \mathrm{~cm}$ map, $\sim 100 \mu \mathrm{Jy}$ to $\sim 35 \mu \mathrm{Jy}$ for the $1.3 \mathrm{~cm}$ data taken on 2014 March 3).

After CLEANing, each map was corrected for attenuation by the primary beam, using the primary beam at the central frequency of each band. The bandwidth of our observations is a significant fraction of the central frequency. However, the primary beam correction may vary significantly over the band. We have computed the error induced in wideband fluxes measured when correcting by the primary beam of the central frequency, rather than the appropriate primary beam for each channel, and find that this error is $<5 \%$ for realistic spectral indices $(-0.1-2)$.

Finally, we restored each map with a CLEAN beam whose size is determined by a Gaussian fit to the central peak of the dirty beam for that map. The size of this beam is given approximately by $\lambda / B_{\max }$ for the map, but the exact size and shape depend on the distribution of baselines in the $u v$-plane and the choice of weighting function. We list the beam sizes for each map in Table 1. After our initial CLEANing of the data, we self-calibrated on the brightest sources in our maps to remove residual beam structure and improve the sensitivity, particularly in crowded regions.

\section{ANALYSIS}

\subsection{Source Detection}

In each of our VLA maps, we search for sources detected above a certain signal-to-noise threshold. Our maps contain 


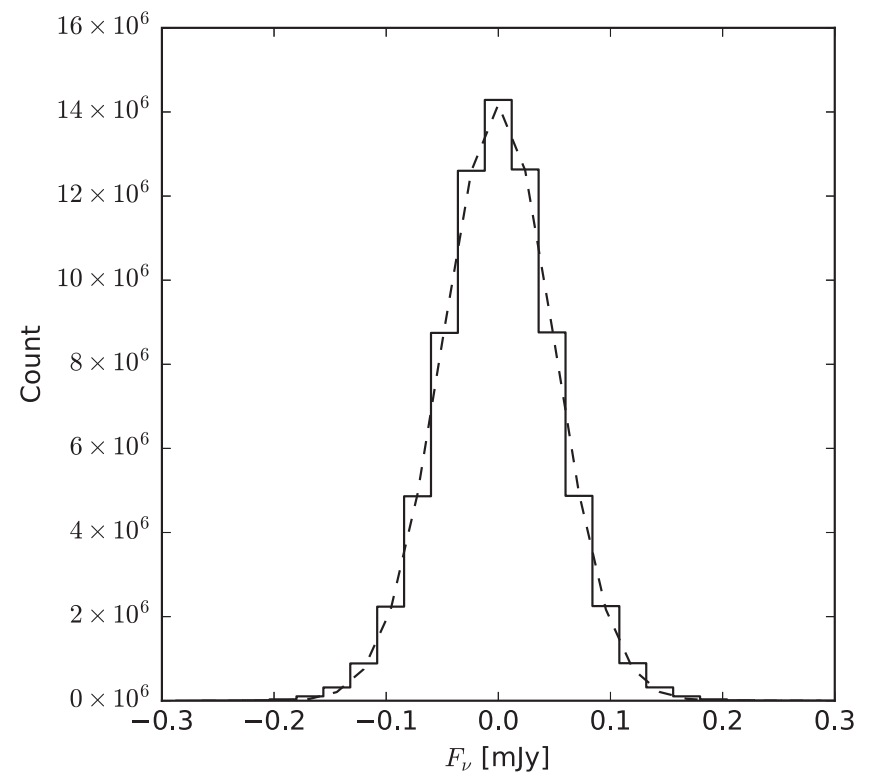

Figure 2. We show a histogram of all the pixel values within the $50 \%$ gain contour of our $6 \mathrm{~cm}$ residual map. We also show the best fit Gaussian to the distribution with the dashed line. Here, we show only the $6 \mathrm{~cm}$ map, but we have produced similar figures for the 3.6 and $1.3 \mathrm{~cm}$ maps and find that both of those distributions are also Gaussian, so that we can use a $\sigma$-cut to confidently distinguish between real sources and noise spikes in our images.

$>10^{6}$ synthesized beams (see Table 1 ), and so we must employ a relatively conservative threshold to ensure that we do not select noise spikes in the images as real detections. The noise in each map follows a Gaussian distribution (see Figure 2), so that we expect $\ll 1$ noise spike to fall above a $6 \sigma$ detection threshold. We, therefore, use $6 \sigma$ as our detection limit.

We can also use catalogs of previously known source positions to target our search. We search our maps at the positions of $>700$ near-infrared-detected sources (Hillenbrand \& Carpenter 2000) and 200 HST-detected proplyds (Ricci et al. 2008, with an overlap of about $\sim 150$ of the near-infrared detected sources). We also search the coordinates of known submillimeter sources detected with the SMA, CARMA, and ALMA that lack counterparts at infrared wavelengths (Eisner et al. 2008; Mann \& Williams 2010; Mann et al. 2014). Finally, we search for compact radio sources, which were detected with the VLA by previous surveys (Felli et al. 1993a; Kounkel et al. 2014). Due to the smaller number of synthesized beams being probed $(\sim 800)$, we expect $\ll 1$ noise spike to fall above a $4.5 \sigma$ level. For each previously identified source, we search for a detection above $4.5 \sigma$ within a radius of $0 . " 5$, typical of the sizes of beams from these previous studies.

The rms at each pixel is calculated from a $128 \times 128$ pixel box surrounding that pixel in the residual map. The rms in the map is generally low $(\sim 25 \mu \mathrm{Jy}$ in the $1.3 \mathrm{~cm}$ maps, $\sim 30 \mu \mathrm{Jy}$ at $3.6 \mathrm{~cm}$, and $\sim 37 \mu \mathrm{Jy}$ at $6 \mathrm{~cm}$ ). However, the central region of each map exhibits beam artifacts from a cluster of bright sources and poor sampling of large-scale emission. The rms in these regions can be much higher than the rest of the map $(\sim 100 \mu \mathrm{Jy}$ in the $1.3 \mathrm{~cm}$ maps, $\sim 70 \mu \mathrm{Jy}$ at $3.6 \mathrm{~cm}$, and $\sim 150 \mu \mathrm{Jy}$ at $6 \mathrm{~cm}$; see Table 1$)$.

We list the total number of sources detected in each map in Table 1. For each map, we also provide a breakdown of the number of sources detected in our blind search as well as the additional number of sources detected from the catalog-driven search. We detect 108 objects in our $6 \mathrm{~cm}$ map, 98 objects in our $3.6 \mathrm{~cm}$ map, and a total of 144 objects across all of our $1.3 \mathrm{~cm}$ maps. In all we detect 175 distinct sources across all of our maps. We show the position of every detected source in our maps in the left panel of Figure 1.

Of the 175 unique compact radio sources, 120 sources are associated with YSOs detected in near-infrared surveys (e.g., Hillenbrand \& Carpenter 2000), and 67 sources are associated with $H S T$-detected proplyds. 149 have previous radio detections, and 40 have been previously detected at millimeter wavelengths. We also report the detection of 11 sources here for the first time at any wavelength.

We fit every detected source with a two-dimensional Gaussian to determine position, extent, and total source flux. For sources identified in the previously mentioned catalogs that are not detected in our maps, we also integrate over a $1^{\prime \prime}$ aperture centered on the known source position to produce an unbiased estimate of the signal (or noise) toward that position. We include a $10 \%$ error on the measurement to account for systematic errors in the band-to-band flux calibration. These intensities, measured toward all cataloged objects in our field of view, are presented in Table 2. The print version of this paper presents only the first page of that table. We also plot images of those sources in Figure 3.

Our catalog of sources, as presented in Table 2, is sorted by right ascension and then given a catalog "ID," which we list in Table 2. We refer to each source by this ID throughout the remainder of the text and figures. In Table 2 we also list the proplyd name, identification from early ONC radio surveys (e.g., Garay et al. 1987; Felli et al. 1993b), identification from Zapata et al. (2004a), or identification from Hillenbrand \& Carpenter (2000), when applicable.

\subsection{Estimating the Free-Free Emission Spectrum}

Evidence suggests that the proplyds are undergoing mass loss from photoevaporation by the nearby $\mathrm{O}$ star $\theta^{1}$ Ori $\mathrm{C}$, so the free-free emission we detect here is likely due to a wind (e.g., Churchwell et al. 1987; Henney \& Arthur 1998). For emission from a spherically symmetric wind with an arbitrary $n \propto r^{-\alpha}$ density profile the expected spectral dependence of free-free emission is

$$
F_{\nu, f f}= \begin{cases}F_{\nu, \text { turn }}\left(\frac{\nu}{\nu_{\text {turn }}}\right)^{-0.1} & \nu \geqslant \nu_{\text {turn }} \\ F_{\nu, \text { turn }}\left(\frac{\nu}{\nu_{\text {turn }}}\right)^{(4 \alpha-6.2) /(2 \alpha-1)} & \nu<\nu_{\text {turn }}\end{cases}
$$

(Wright \& Barlow 1975). $\nu_{\text {turn }}$ is the frequency where the wind becomes partially optically thick, and is determined by the radius of the inner boundary of the ionized envelope. High turnover frequencies indicate more compact inner boundaries. When the wind becomes fully optically thick at very low frequencies the spectrum follows the typical $F_{\nu} \propto \nu^{2}$ spectrum expected for optically thick thermal emission.

For a fully ionized wind with a constant mass-loss rate, we expect $\alpha=2$ and the spectral dependence of free-free 
Table 2

Source Detections, Identifications, and Fluxes

\begin{tabular}{|c|c|c|c|c|c|c|c|c|c|c|c|c|c|c|}
\hline ID & $\begin{array}{l}\text { Proplyd } \\
\text { Name }\end{array}$ & HC00 ID & GMR ID & Z04a ID & $\begin{array}{l}\text { Other } \\
\text { Names }\end{array}$ & $\begin{array}{l}\text { R.A. } \\
\text { (J2000) }\end{array}$ & $\begin{array}{l}\text { Decl. } \\
\text { (J2000) }\end{array}$ & $\begin{array}{l}F_{\nu, 6 \mathrm{~cm}} \\
(\mathrm{mJy})\end{array}$ & $\begin{array}{c}F_{\nu, 3.6 \mathrm{~cm}} \\
(\mathrm{mJy})\end{array}$ & $\begin{array}{c}F_{\nu, 1.3 \mathrm{~cm}, 1^{\mathrm{a}}} \\
\quad(\mathrm{mJy})\end{array}$ & $\begin{array}{c}F_{\nu, 1.3 \mathrm{~cm}, 2^{\mathrm{a}}} \\
\quad(\mathrm{mJy})\end{array}$ & $\begin{array}{c}F_{\nu, 1.3 \mathrm{~cm}, 3^{\mathrm{a}}} \\
(\mathrm{mJy})\end{array}$ & $\begin{array}{c}F_{\nu, 1.3 \mathrm{~cm}, 4^{\mathrm{a}}} \\
(\mathrm{mJy})\end{array}$ & $\begin{array}{c}F_{\nu, 1.3 \mathrm{~cm}, \text { mean }}{ }^{\mathrm{a}} \\
(\mathrm{mJy})\end{array}$ \\
\hline 1 & $4538-311$ & $\ldots$ & $\ldots$ & $\ldots$ & $\ldots$ & $5^{\mathrm{h}} 34^{\mathrm{m}} 53^{\mathrm{s}} .79$ & $-5^{\mathrm{d}} 23^{\mathrm{m}} 10^{\mathrm{s}} .73$ & $0.015 \pm 0.567$ & $\ldots$ & $\ldots$ & $\ldots$ & $\ldots$ & $\ldots$ & $\ldots$ \\
\hline 2 & $\ldots$ & $\ldots$ & $\ldots$ & $\ldots$ & $\ldots$ & $5^{\mathrm{h}} 34^{\mathrm{m}} 55^{\mathrm{s}} .97$ & $-5^{\mathrm{d}} 23^{\mathrm{m}} 13^{\mathrm{s}} .00$ & $0.269 \pm 0.338$ & $\ldots$ & $\ldots$ & $\ldots$ & $\ldots$ & $\ldots$ & $\ldots$ \\
\hline 3 & $4582-635$ & $\ldots$ & $\ldots$ & $\ldots$ & $\ldots$ & $5^{\mathrm{h}} 34^{\mathrm{m}} 58^{\mathrm{s}} \cdot 16$ & $-5^{\mathrm{d}} 26^{\mathrm{m}} 35^{\mathrm{s}} .13$ & $-0.245 \pm 0.409$ & $\ldots$ & $\ldots$ & $\ldots$ & $\ldots$ & $\ldots$ & $\ldots$ \\
\hline 4 & $005-514$ & $\ldots$ & $\ldots$ & $\ldots$ & $\ldots$ & $5^{\mathrm{h}} 35^{\mathrm{m}} 00^{\mathrm{s}} \cdot 47$ & $-5^{\mathrm{d}} 25^{\mathrm{m}} 14^{\mathrm{s}} .34$ & $0.114 \pm 0.233$ & $\ldots$ & $\ldots$ & $\ldots$ & $\ldots$ & $\ldots$ & $\ldots$ \\
\hline 5 & $006-439$ & $\ldots$ & $\ldots$ & $\ldots$ & $\ldots$ & $5^{\mathrm{h}} 35^{\mathrm{m}} 00^{\mathrm{s}} .58$ & $-5^{\mathrm{d}} 24^{\mathrm{m}} 38^{\mathrm{s}} .79$ & $-0.008 \pm 0.229$ & $-0.049 \pm 1.203$ & $\ldots$ & $\ldots$ & $\ldots$ & $\ldots$ & $\ldots$ \\
\hline
\end{tabular}

Note.

${ }^{\mathrm{a}}$ Subscript 1 indicates the $1.3 \mathrm{~cm}$ data were taken on 2013 November 10, 2 on 2014 March 3, 3 on 2014 March 7, 4 on 2014 May 3 , and the mean indicates the image from the combined $1.3 \mathrm{~cm}$ tracks.

(This table is available in its entirety in machine-readable form.) 

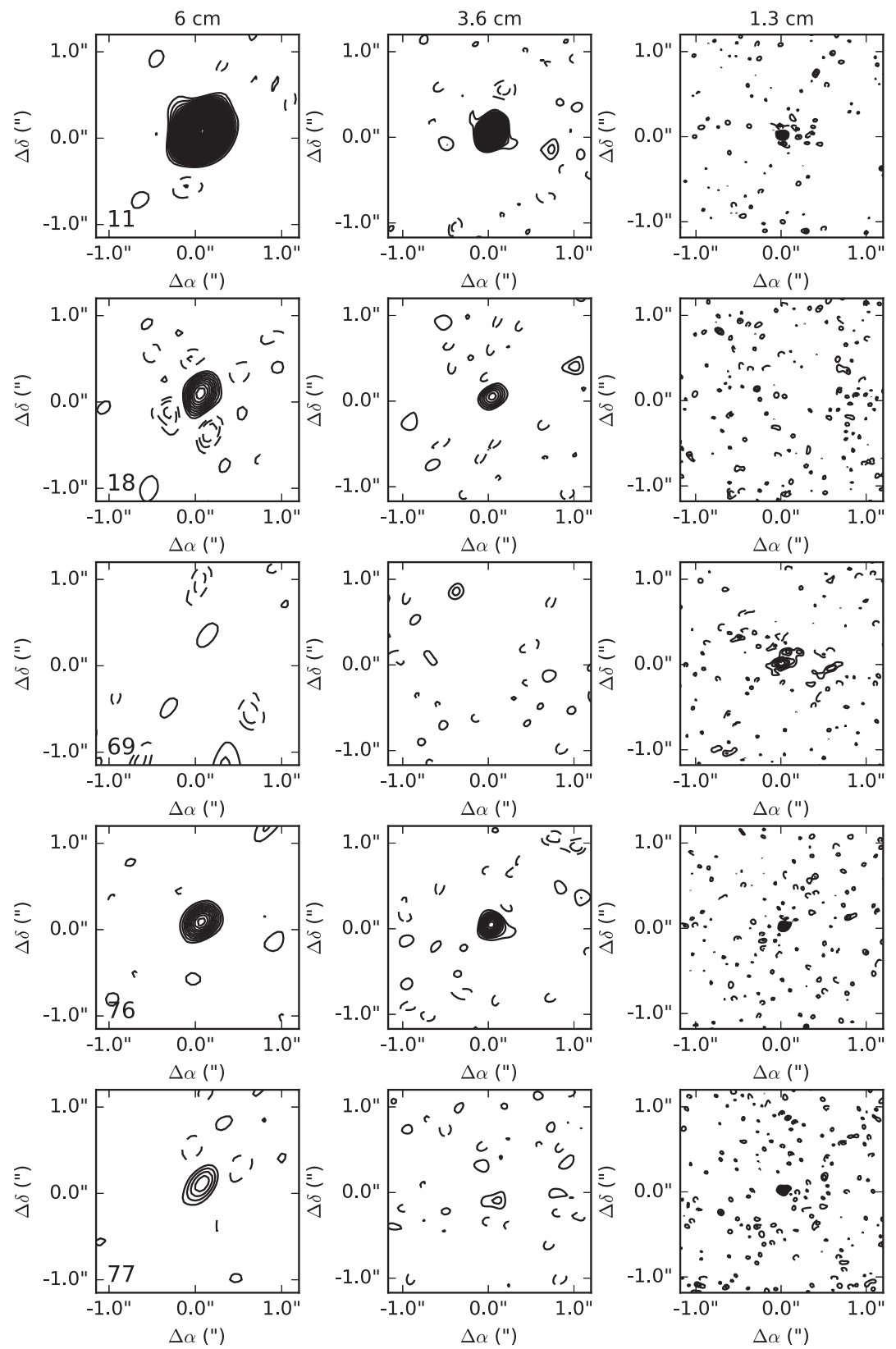

Figure 3. Contour images of sources detected in our $6 \mathrm{~cm}, 3.6 \mathrm{~cm}$ or $1.3 \mathrm{~cm}$ continuum maps. Each row shows a single source in each band. At $1.3 \mathrm{~cm}$, we show only one epoch as a representative image of the source. Contour increments are $1 \sigma$, beginning at $\pm 2 \sigma$, where $\sigma$ is determined locally for each object.

(An extended version of this figure is available.)

emission is

$$
F_{\nu, f f}=\left\{\begin{array}{l}
F_{\nu, \text { turn }}\left(\frac{\nu}{\nu_{\text {turn }}}\right)^{-0.1} \quad \nu \geqslant \nu_{\text {turn }} \\
F_{\nu, \text { turn }}\left(\frac{\nu}{\nu_{\text {turn }}}\right)^{0.6} \quad \nu<\nu_{\text {turn }}
\end{array} .\right.
$$

Steeper density profiles may lead to steeper spectral dependences below the turnover frequency (e.g., Plambeck et al. 1995). Here, for simplicity, we adopt the solution for a fully ionized wind with a constant mass-loss rate. Many of our sources show evidence for a free-free turnover (see Figure 4), and so we adopt a model including a turnover in the spectrum.
At higher frequencies, dust emission is expected to dominate. The differences in the expected spectral slopes between dust and free-free emission allow us to characterize each separately by observing our targets at a range of wavelengths. For each of our detected sources we fit a simple model to the known radio, millimeter, and submillimeter photometry:

$$
F_{\nu}=F_{\nu, f f}+F_{\nu, \text { dust }, 230 \mathrm{GHz}}\left(\frac{\nu}{230 \mathrm{GHz}}\right)^{2+\beta}
$$

Here, we assume $\beta=0.7$, consistent with previous studies of protoplanetary disks in other star-forming regions (e.g., Rodmann et al. 2006; Ricci et al. 2010a, 2010b). 

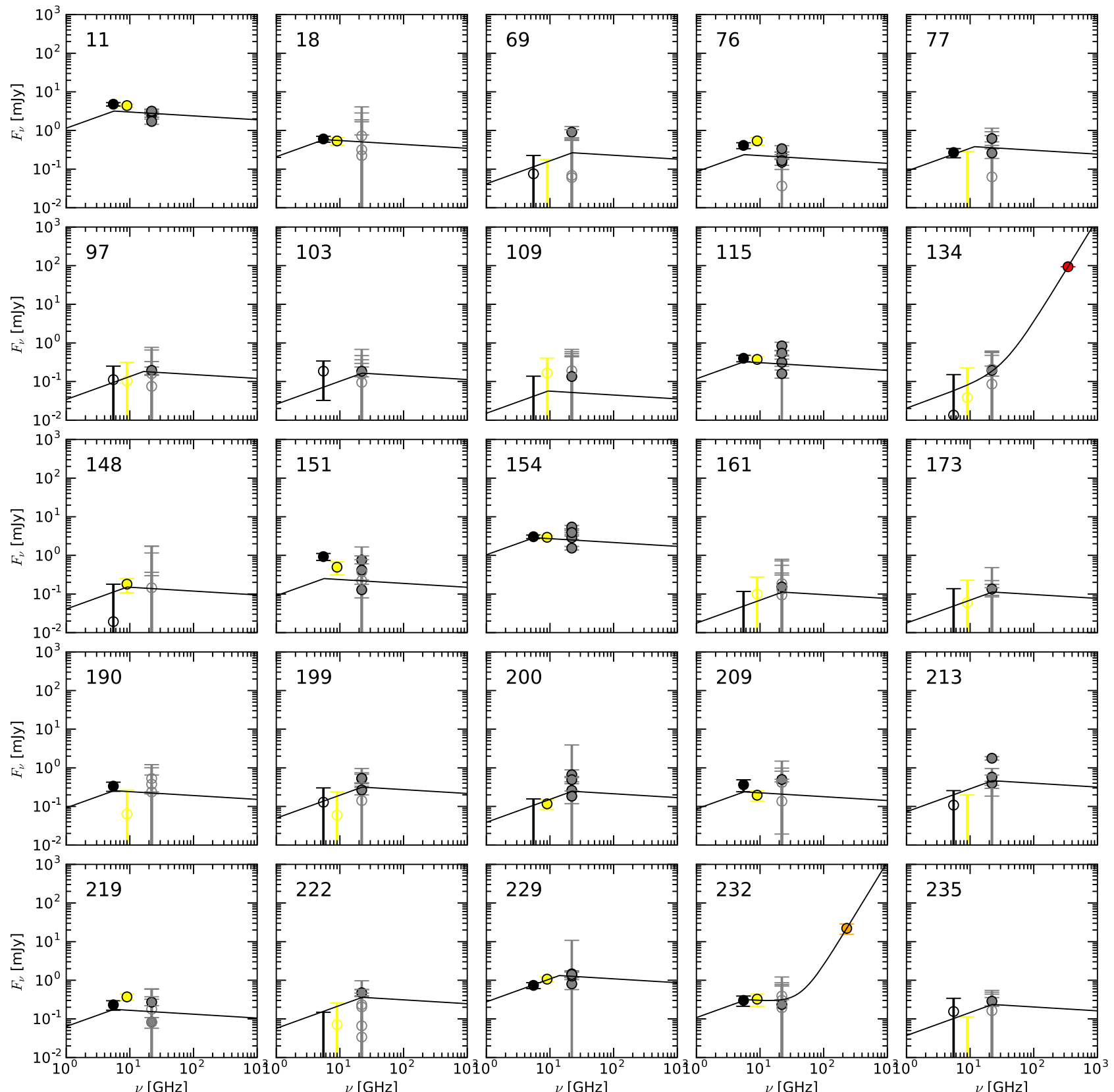

Figure 4. The millimeter and radio spectral energy distributions (SEDs) for all of the sources detected in our maps. We also show the best fit dust + free-free emission model for each source, as described in Section 3.2. Black, yellow and gray points are the 6,3.6, and $1.3 \mathrm{~cm}$ flux measurements for objects detected in our maps. Circles with colored faces indicate that the source was detected by our search routines, while open face circles are fluxes measured in an aperture around a known source position. Orange data points are 3, $1.3 \mathrm{~mm}$, and $870 \mu \mathrm{m}$ fluxes from Eisner et al. (2008, and references therein). Green data points are $870 \mu \mathrm{m}$ fluxes from Mann \& Williams (2010), and red data points are $870 \mu \mathrm{m}$ fluxes from Mann et al. (2014). The fluxes shown here are all measured with one of the SMA, CARMA, ALMA, OVRO, or the VLA.

(An extended version of this figure is available.)

We fit the SED of each source by searching a grid over a large range of parameter space of $\nu_{\mathrm{turn}}, F_{\nu, \text { turn }}$, and $F_{\nu \text {,dust, } 230 \mathrm{GHz}}$ for a minimum in $\chi^{2}$. We then use a second, finely spaced, grid search based on the initial search to find the best $\chi^{2}$ fit.

$\nu_{\text {turn }}, F_{\nu, \text { turn }}$ and $F_{\nu, \text { dust, } 230 \mathrm{GHz}}$ are left as free parameters in the grid search. If a source has no submillimeter detections ( $\geqslant 90 \mathrm{GHz}$; Eisner et al. 2008; Mann \& Williams 2010; Mann et al. 2014), we assume that $F_{\nu, \text { dust, } 230 \mathrm{GHz}}=0$. In that case, we also require $5.5 \mathrm{GHz} \leqslant \nu_{\text {turn }} \leqslant 22 \mathrm{GHz}$, because outside of this range we cannot constrain $\nu_{\text {turn }}$. If a source does have submillimeter detections, we only require $5.5 \mathrm{GHz} \leqslant \nu_{\text {turn }}$.

Sources 281, 391, 416, 423, 430, 433, 442, 512, 516, 537, 564 , and 595 are all extended sources that are marginally resolved by our 3.6 and $6 \mathrm{~cm}$ maps, as well as in our B-configuration $1.3 \mathrm{~cm}$ observations. In our A-configuration $1.3 \mathrm{~cm}$ observations, however, these sources are very well resolved. In fact, they are so well resolved that much or all of the emission from the source is resolved out. As such, we exclude the A-configuration flux measurements from our SED 
fitting, as flux variations are likely due to structure being resolved out rather than actual variability.

Some of our sources are variable across our multiple epochs of $1.3 \mathrm{~cm}$ data (see Section 4.2). We account for this variability in our modeling by including the measured flux at each epoch and allowing the variability to influence the uncertainty of our parameter estimation. Sources that are more variable will also have more uncertainty in model fits.

We list the parameters of our best fitted models to each source detected in our maps in Table 3 . The photometry, along with the best fit model, for each source is plotted in Figure 4.

The origin of the radiation ionizing these sources has been the subject of much debate. Early radio studies of the region disagreed as to whether these sources were externally ionized by radiation from $\theta^{1}$ Ori $\mathrm{C}$ or ionized internally by a young massive star, and as to whether these objects are dense neutral condensations or protoplanetary disks (e.g., Churchwell et al. 1987; Garay et al. 1987), although these studies are complicated by the fact that only projected, and not actual, distances from $\theta^{1}$ Ori $\mathrm{C}$ are known. Since these early studies, HST imaging (e.g., O'Dell et al. 1993) and detailed modeling of those images (e.g., Henney \& Arthur 1998) has favored protoplanetary disks ionized by $\theta^{1}$ Ori C.

Free-free emission powered by ionizing radiation from $\theta^{1}$ Ori $\mathrm{C}$ should decrease with increasing separation from $\theta^{1}$ Ori $\mathrm{C}$. We show the measured flux versus distance in the left panel of Figure 5. For most sources there is a trend of decreasing radio flux with increasing separation, suggesting that they are exhibiting free-free emission from gas ionized by $\theta^{1}$ Ori $\mathrm{C}$. We also find that the difference in their 1.3 and $6 \mathrm{~cm}$ flux is close to zero, as expected for optically thin free-free emission, which has a roughly flat spectrum (see the right panel of Figure 5). We note that here we report projected distances. Actual separations are greater than or equal to this number. We discuss the outliers of these trends below, in Section 4.3.

For this study, we are only concerned with whether these sources are emitting thermal free-free emission or not, so that we can characterize the emission and remove it from dust emission for disk mass studies, but on the surface Figure 5 would seem to suggest that these sources are externally ionized by $\theta^{1}$ Ori $\mathrm{C}$. The detailed structure of these compact objects is beyond the scope of this paper, as the radio emission can be well characterized without that knowledge, but we will revisit the subject in a more detailed study in the future.

\section{DISCUSSION}

\subsection{Comparison with Previous Radio Surveys}

Many compact radio sources have previously been identified at a range of wavelengths in the ONC through VLA surveys of the region. The earliest searches for compact radio sources in the ONC were conducted primarily at 20,6,2, and $1.3 \mathrm{~cm}$ (e.g., Churchwell et al. 1987; Garay et al. 1987; Felli et al. 1993a, 1993b). These surveys were state of the art at the time, with rms as low as $0.18 \mathrm{mJy} \mathrm{beam}^{-1}$ at $2 \mathrm{~cm}$ (Churchwell et al. 1987; Felli et al. 1993a), $0.23 \mathrm{mJy}$ beam $^{-1}$ at $6 \mathrm{~cm}$ (Felli et al. 1993b), or $1.0 \mathrm{mJy}^{\text {beam }}{ }^{-1}$ at $1.3 \mathrm{~cm}$ (Garay et al. 1987). These searches identified 49 compact radio sources in the ONC.

A more recent survey mapped a $4^{\prime} \times 4^{\prime}$ region of the the ONC at $3.6 \mathrm{~cm}$ using the VLA. This survey achieved a sensitivity of $0.03 \mathrm{mJy}$ beam $^{-1}$ and uncovered 77 compact radio sources (Zapata et al. 2004a). Of these 77 sources, 38 were previously known from the earlier studies mentioned above, while 39 were new centimeter detections. Zapata et al. (2004b) also mapped a $30^{\prime \prime} \times 30^{\prime \prime}$ region in OMC-1 South at $1.3 \mathrm{~cm}$ with the VLA. They achieved an $\mathrm{rms}$ of $0.07 \mathrm{mJy}$ beam $^{-1}$, but due to the limited area of their maps only detected 11 sources.

A recent survey mapped out a large region encompassing $\lambda$ Ori, Lynds 1622, NGC 2068, NGC 2071, NGC 2023, NGC 2024, $\sigma$ Ori, the ONC, and Lynds 1641 with the VLA at 4.5 and $7.5 \mathrm{GHz}$ with a $60 \mu \mathrm{Jy}$ sensitivity (Kounkel et al. 2014). They found $>350$ sources over the area of their map, 54 of which overlap with the area we survey. The majority of their detected sources also have spectral indices consistent with flat spectra.

Here, we compare these previous surveys with our own JVLA maps. In Figure 6 we plot the distribution of fluxes for compact sources detected in our maps as well as the distribution of fluxes for previously identified compact radio sources at the same wavelength. The most extensive existing studies at $1.3 \mathrm{~cm}$ are limited by either survey area or sensitivity so we also compare our $1.3 \mathrm{~cm}$ detections with previous $2 \mathrm{~cm}$ detections.

Of the 49 compact radio sources detected by initial surveys (e.g., Churchwell et al. 1987; Garay et al. 1987; Felli et al. 1993a, 1993b), we have detected 37 in our maps. We have also recovered 64 of the 77 sources detected by Zapata et al. (2004a), 9 of the 11 sources found by Zapata et al. (2004b), 42 of the 54 sources found by Kounkel et al. (2014), and 144 of the 556 sources found by Forbrich et al. (2016). We detect 29 of the 35 sources that were previously detected at $2 \mathrm{~cm}$. We also report the detection of 135 sources that have not previously been detected at $1.3 \mathrm{~cm}, 34$ at $3.6 \mathrm{~cm}, 4$ at $6 \mathrm{~cm}$, and 26 sources that have not previously been detected at any radio wavelengths. The sources that were previously detected, but that we do not detect in our maps, are likely variable given the deeper sensitivity in our JVLA data.

\subsection{Variability}

Previous radio studies of the ONC have explored multiple epochs of data to search for evidence of source variability. Felli et al. (1993b) monitored the ONC at 2 and $6 \mathrm{~cm}$ for a period of 7 months and found 13 sources to be variable over that time with flux variability of $20 \%-80 \%$. Zapata et al. (2004a) tracked the ONC at $3.6 \mathrm{~cm}$ over 4 years, and identified 36 sources that are time variable by more than $30 \%$. More recently, Kounkel et al. (2014) mapped a large region of the Gould Belt at 4.5 and $7.5 \mathrm{GHz}$ over three epochs each separated by a month, and found 32 variable sources in the ONC. Furthermore, Rivilla et al. (2015) studied a field in the ONC at 0.7 and $0.9 \mathrm{~cm}$ and found 19 sources which are variable over long-term (monthly) timescales, and five sources which are variable on short timescales (hours to days). Moreover, very short timescale radio flares have been observed toward a number of pre-main sequence stars (e.g., Bower et al. 2003; Forbrich et al. 2008; Rivilla et al. 2015)

Here, we compare previously measured fluxes for detected compact radio sources with the fluxes in our maps. Timebaselines are $\sim 10$ years at $1.3 \mathrm{~cm}$ and $3.6 \mathrm{~cm}$ and $\gtrsim 20$ years at $6 \mathrm{~cm}$, and we cannot characterize shorter timescales for variability. We thus seek to identify sources that may not have been detected as variable in previous, shorter time-baseline 
Table 3

Free-Free Emission Model Parameters and ALMA Band Fluxes for Detected Sources

\begin{tabular}{|c|c|c|c|c|c|c|c|c|c|c|c|c|c|}
\hline ID & $\begin{array}{c}\nu_{\text {turn }} \\
(\mathrm{GHz})\end{array}$ & $\begin{array}{l}F_{\nu, \text { turn }} \\
(\mathrm{mJy})\end{array}$ & $\begin{array}{c}F_{\nu, \text { dust, } 230 \mathrm{GHz}} \\
(\mathrm{mJy})\end{array}$ & $\begin{array}{c}F_{\nu, \text { Band } 1}{ }^{\mathrm{a}} \\
(\mathrm{mJy})\end{array}$ & $\begin{array}{c}F_{\nu, \text { Band } 2}{ }^{\mathrm{a}} \\
(\mathrm{mJy})\end{array}$ & $\begin{array}{c}F_{\nu, \text { Band }} 3^{\mathrm{a}} \\
(\mathrm{mJy})\end{array}$ & $\begin{array}{c}F_{\nu, \text { Band } 4^{\mathrm{a}}} \\
(\mathrm{mJy})\end{array}$ & $\begin{array}{c}F_{\nu, \text { Band } 5}{ }^{\mathrm{a}} \\
(\mathrm{mJy})\end{array}$ & $\begin{array}{c}F_{\nu, \text { Band } 6}{ }^{\mathrm{a}} \\
(\mathrm{mJy})\end{array}$ & $\begin{array}{c}F_{\nu, \text { Band }} 7^{\mathrm{a}} \\
(\mathrm{mJy})\end{array}$ & $\begin{array}{c}F_{\nu, \text { Band } 8}{ }^{\mathrm{a}} \\
(\mathrm{mJy})\end{array}$ & $\begin{array}{c}F_{\nu, \text { Band } 9}{ }^{\mathrm{a}} \\
(\mathrm{mJy})\end{array}$ & $\begin{array}{c}F_{\nu, \text { Band } 10}{ }^{\mathrm{a}} \\
(\mathrm{mJy})\end{array}$ \\
\hline 11 & $<5.5$ & $3.188 \pm 0.161$ & $\cdots$ & 2.65 & 2.44 & 2.39 & 2.29 & 2.23 & 2.19 & 2.11 & 2.05 & 1.98 & 1.93 \\
\hline 18 & $<5.5$ & $0.582 \pm 0.078$ & $\ldots$ & 0.48 & 0.45 & 0.44 & 0.42 & 0.41 & 0.40 & 0.38 & 0.37 & 0.36 & 0.35 \\
\hline 69 & $>22.0$ & $0.265 \pm 0.181$ & $\cdots$ & 0.25 & 0.23 & 0.23 & 0.22 & 0.21 & 0.21 & 0.20 & 0.20 & 0.19 & 0.18 \\
\hline 76 & $<5.5$ & $0.237 \pm 0.021$ & $\ldots$ & 0.20 & 0.18 & 0.18 & 0.17 & 0.17 & 0.16 & 0.16 & 0.15 & 0.15 & 0.14 \\
\hline 77 & $11.477 \pm 6.002$ & $0.382 \pm 0.055$ & $\ldots$ & 0.34 & 0.31 & 0.31 & 0.30 & 0.29 & 0.28 & 0.27 & 0.26 & 0.25 & 0.25 \\
\hline
\end{tabular}

Note.

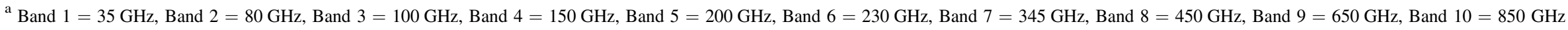

(This table is available in its entirety in machine-readable form.) 

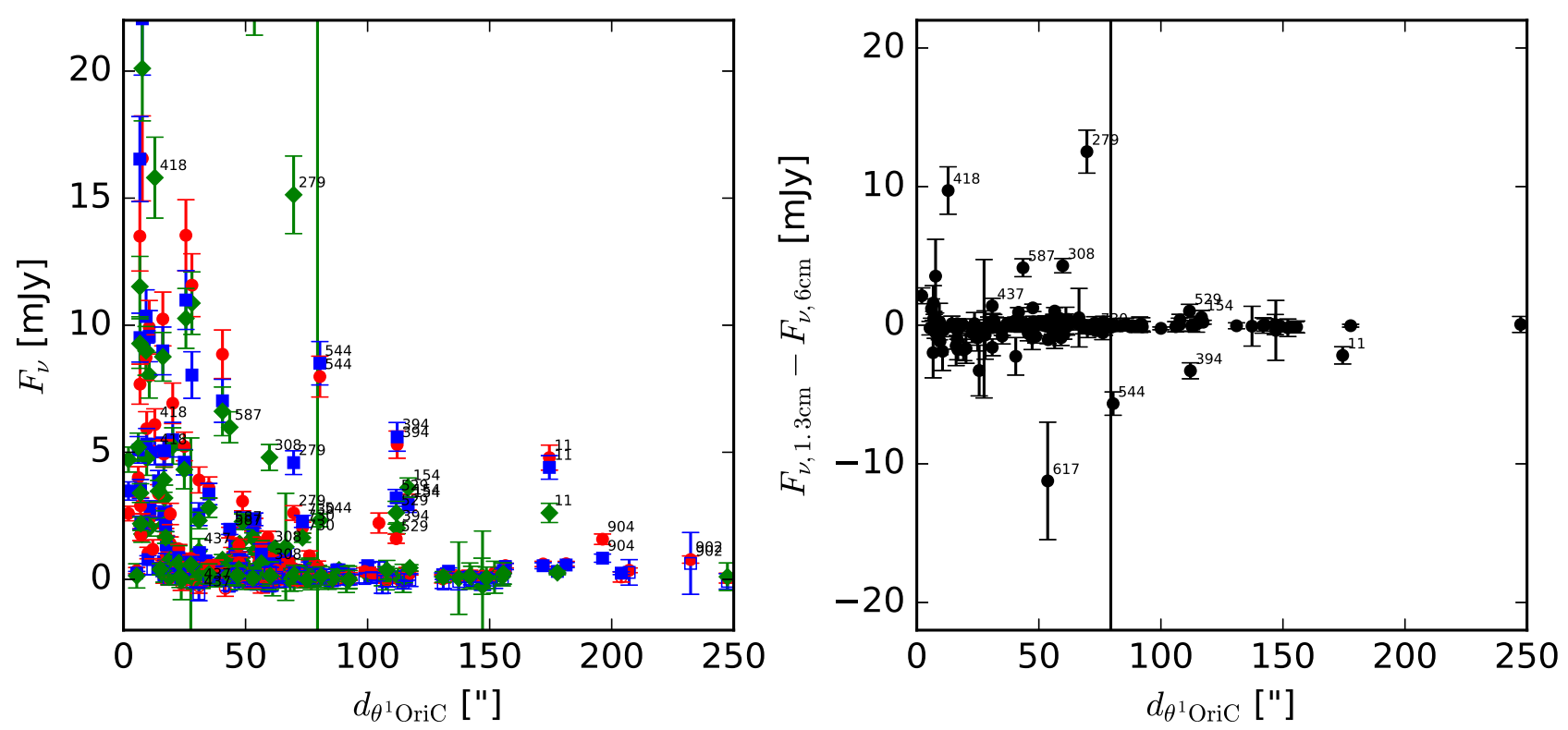

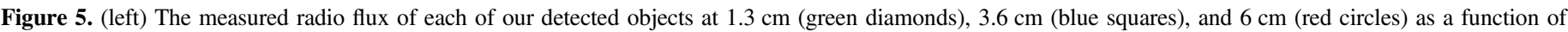

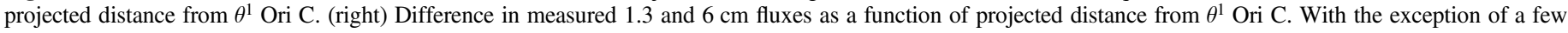

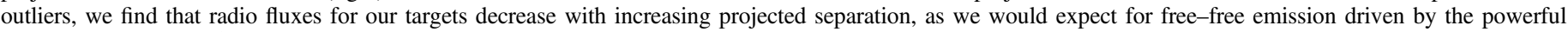

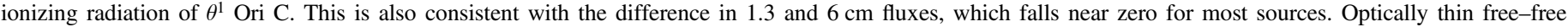

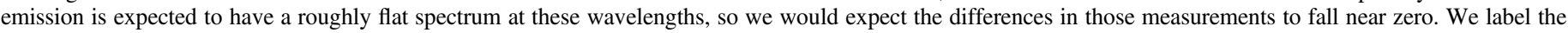
significant outliers with the source ID for reference in future sections.

studies (Felli et al. 1993b; Zapata et al. 2004a). We also use our multiple epochs of $1.3 \mathrm{~cm}$ data to search for variability on timescales of $\sim 7$ months, between 2013 November 10 and 2014 May 3.

As discussed earlier, Sources 281, 391, 416, 423, 430, 433, $442,512,516,537,564$, and 595 are very well resolved with the A-configuration at $1.3 \mathrm{~cm}$. As such, we exclude these sources from our variability considerations at $1.3 \mathrm{~cm}$, as flux variations may be due to structure being resolved out rather than actual variability.

We define a variable source as one for which the flux measurements are $3 \sigma$ discrepant from one epoch to the next, at any observed wavelength. $\Delta F / F$ quantifies how variable a source is, where $F$ is the mean flux of the source and $\Delta \mathrm{F}$ is the standard deviation of the fluxes. We show the results of this search in Table 4. For the sources detected in Zapata et al. (2004a) and Zapata et al. (2004b) we include a 10\% uncertainty on the flux on top of the uncertainties they quote to account for a systematic flux calibration uncertainty across the data sets.

At $1.3 \mathrm{~cm}$, we find 30 sources that show some indication of variability, with $\Delta F / F$ ranging from $20 \%-900 \%$. At $3.6 \mathrm{~cm}$, we identify 32 sources whose fluxes are variable, including three sources not identified as variable in Zapata et al. (2004a), because they were too faint to be detected in individual epochs. The variability, as defined by $\Delta F / F$, of these sources ranges from $20 \%-200 \%$. Finally, at $6 \mathrm{~cm}$ we identify five variable sources with $\Delta F / F$ ranging from $50 \%-100 \%$.

There were 13 sources detected by previous radio surveys of the ONC (e.g., Felli et al. 1993a, 1993b). Most of those sources were not detected in the same bands as our observations, and so they are excluded from our variability analysis. However, the five variable sources with $6 \mathrm{~cm}$ fluxes from Felli et al. (1993b) were undetected in our maps, and have $\Delta F / F$ ranging from $50 \%-100 \%$. Given the high fluxes of the remainder of the sources, we would have expected to detect them in our maps, and so those sources likely have similarly high variability amplitudes.

In all, we find that 55 of our sources are variable at one or more wavelengths. Of the variable sources, 11 are characterized as variable at multiple wavelengths. 20 are found to be variable at one wavelength, but not another, although many of our constraints on $\Delta F / F$ are not strong. The remaining sources could only be analyzed at a single wavelength.

We show the location of each variable source in the right panel of Figure 1, with the strength of the variability $(\Delta F / F)$ represented by the size of the plot symbol. We find that variability amplitude does not follow the same trend as freefree flux, with variability decreasing with increased separation from $\theta^{1}$ Ori $\mathrm{C}$. Instead, we find sources that are significantly variable out to large radii. Some of the most variable objects can be found at large separations.

Variability of radio emission from these sources is likely to arise from a few different mechanisms. It may be the result of gyrosynchrotron emission produced by magnetospheric activity in young stars (e.g., Feigelson \& Montmerle 1999). These flares may be the result of magnetic reconnections on the protostellar surface, which would produce radio flares on the timescales of minutes (e.g., Dulk 1985; Bower et al. 2003; Forbrich et al. 2008). Interactions between the magnetic fields of the protostar and its disk could also produce flares on the timescales similar to the rotation periods of young stars, which are typically days to weeks in the ONC (e.g., Shu et al. 1997; Forbrich et al. 2006; Rodríguez-Ledesma et al. 2009).

Free-free emission may also be variable if the density distribution of material being ionized is non-uniform, causing the amount of ionized material to vary, or if the incident ionizing radiation varies. Studies have found that O-type stars have winds that exhibit cyclical variability on timescales of hours to days (see review by Fullerton 2003). The visible, UV and X-ray intensity of $\theta^{1}$ Ori $\mathrm{C}$ varies with a period of 15.4 days 

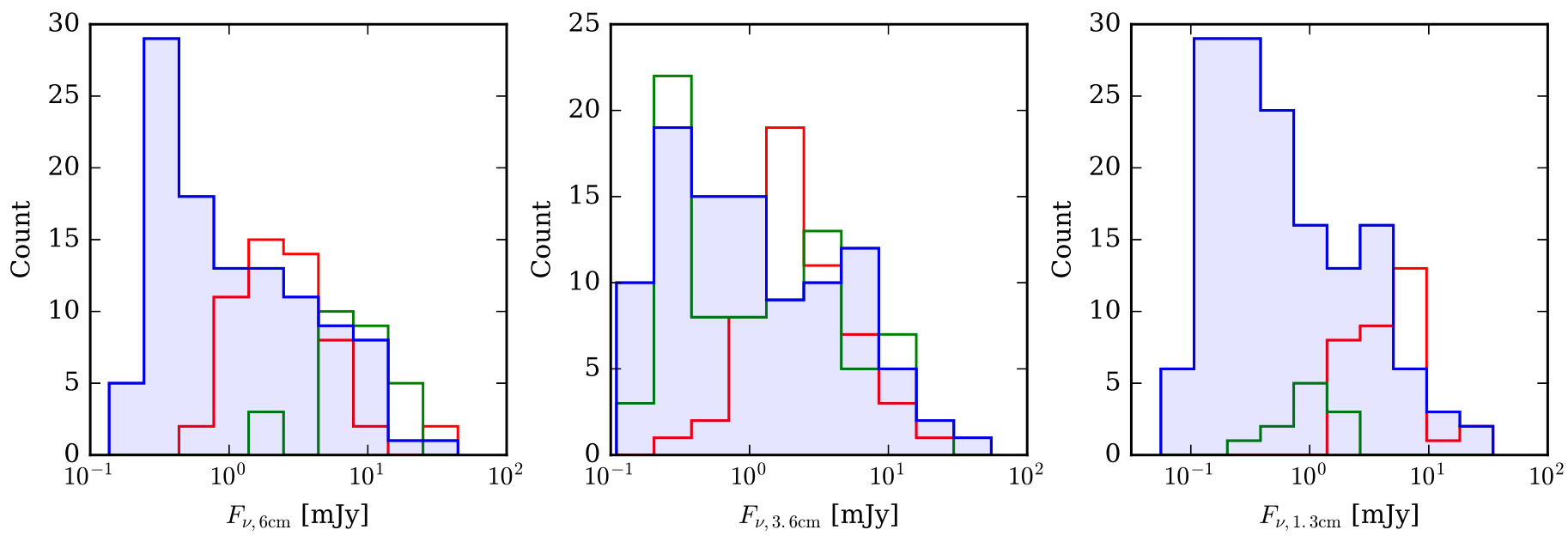

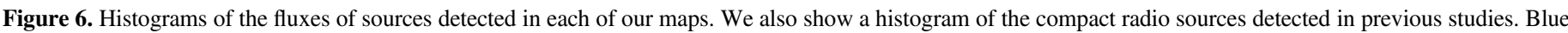

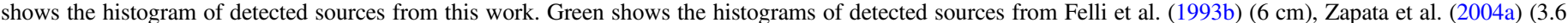

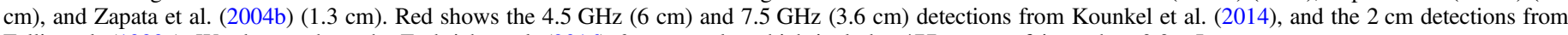
Felli et al. (1993a). We do not show the Forbrich et al. (2016) $6 \mathrm{~cm}$ sample, which includes 477 sources fainter than $0.3 \mathrm{mJy}$.

(e.g., Stahl et al. 1993, 1996; Caillault et al. 1994; Walborn \& Nichols 1994), and so the ionization level and, therefore, freefree flux might be expected to vary on a similar timescale. The ionized region, however, is likely to be many light days across or larger, and so this variability may be washed out.

Inhomogeneities in the disk are unlikely to be brought into the ionized region on timescales shorter than the dynamical timescale. For disks, the dynamical timescale varies depending on location in the disk and the mass of the central star (e.g., Kenyon 2001). Inner disk radii for young stars are found to be in the order of 0.1-1 AU (e.g., Eisner et al. 2007), so that the smallest dynamical timescales we can expect are in the order of weeks to half a year. Photoevaporation in disks tends to produce winds at radii larger than the critical radius, where the photoionized material has sufficient velocity to escape. For ionization by EUV photons, this tends to occur at radii of $\gtrsim 5$ au (e.g., Hollenbach et al. 1994; Gorti \& Hollenbach 2009), corresponding to dynamical timescales of a few years. Nonuniformities in the disk are, therefore, likely to cause longerterm variability in the free-free emission.

Aside from the timescale of variability, the SED of the source at each epoch might be used to distinguish between free-free and synchrotron emission. As described in Section 3.2, free-free emission is characterized by a flat spectrum with $F_{\nu} \propto \nu^{-0.1}$. Gyrosynchrotron emission, however, is expected to have a spectral index that is significantly negative, typically $F_{\nu} \propto \nu^{-0.7}$. We discuss constraints on the nature of some of these sources in Section 4.3. Further studies with concurrent flux measurements at multiple wavelengths, however, are needed to fully distinguish between these sources of emission.

Here, we do not have simultaneous flux measurements at all bands for each epoch of data, and so it is difficult to constrain the spectral index of the emission at each epoch. There are, however, a few sources which change flux significantly between the $1.3 \mathrm{~cm}$ observations on 2014 March 3 and 7. For example, on March 3, Source 529 had a $1.3 \mathrm{~cm}$ flux of $7 \mathrm{mJy}$, but on March 7 it was down to a flux of $4.5 \mathrm{mJy}$. By May 7th the flux was all the way down at $0.5 \mathrm{mJy}$. Such an extreme change in flux may be indicative of gyrosynchrotron emission from a magnetic flare. Source 544 also shows a similar pattern.
Source 587 has a flux of 1.7 mJy on March 3, but on March 7 its flux increased significantly to $22 \mathrm{mJy}$, again possibly indicative of a magnetic flare. For most sources, however, we do not have sufficient time resolution to distinguish between daily, weekly, or even longer variability timescales.

\subsection{Nature of Detected Sources}

We detected emission in at least one of our maps from 67 HST-identified proplyds (Ricci et al. 2008; Mann \& Williams 2010; Mann et al. 2014). Furthermore, we have detected radio emission toward 120 sources that have been identified by nearinfrared imaging (Hillenbrand \& Carpenter 2000). We also detect radio emission from two sources dubbed "MM" by Eisner et al. (2008), indicating that they have previously only been detected at wavelengths longer than $1 \mathrm{~mm}$. Finally, we have detected 51 sources that are not associated with a known proplyd or near-infrared detected source.

The majority of our targets, including all of the sources identified as proplyds, are well fitted by our free-free and dust emission model, in agreement with previous conclusions that these objects are disks with winds driven by photoevaporation (e.g., Churchwell et al. 1987; Henney \& Arthur 1998). We detect a turnover in the free-free emission spectrum for 40 objects, as evidenced by $5.5 \mathrm{GHz}<\nu_{\text {turn }}<22 \mathrm{GHz}$. There are at least three sources (Sources 374, 465, 473) that might even have $\nu_{\text {turn }}>22 \mathrm{GHz}$, indicating that our maps are insufficient to fully characterize their emission. With such high turnover frequencies, these objects must have small inner boundaries to their ionized envelopes and are likely very compact and dense. Further short wavelength observations are necessary to better constrain the free-free emission spectrum.

Some sources have SEDs that appear to be fitted well by our free-free + dust model with some variability included. Fluxes at all three wavelengths were measured concurrently on 2014 March 3, and if we just consider those flux measurements, all of our sources are fitted well by free-free emission models. Without simultaneous 3.6 and $6 \mathrm{~cm}$ measurements for the other $1.3 \mathrm{~cm}$ epochs it is impossible to say whether the SEDs at those epochs remain consistent with free-free emission, although it seems probable. 
Table 4

Variability of ONC Sources

\begin{tabular}{|c|c|c|c|c|c|c|}
\hline ID & $1.3 \mathrm{~cm}$ Variable? & $(\Delta F / F)_{1.3 \mathrm{~cm}}$ & $3.6 \mathrm{~cm}$ Variable? & $(\Delta F / F)_{3.6 \mathrm{~cm}}$ & $6 \mathrm{~cm}$ Variable? & $(\Delta F / F)_{6 \mathrm{~cm}}$ \\
\hline 11 & $\mathrm{~N}$ & $\ldots$ & $\mathrm{Y}$ & $20 \pm 28$ & $\ldots$ & $\ldots$ \\
\hline 76 & $\mathrm{~N}$ & $\ldots$ & $\ldots$ & $\ldots$ & 0 & $\cdots$ \\
\hline 77 & $\mathrm{~N}$ & $\ldots$ & $\mathrm{Y}$ & $144 \pm 25$ & $\ldots$ & $\ldots$ \\
\hline 102 & $\cdots$ & $\cdots$ & $\mathrm{N}$ & $\ldots$ & $\cdots$ & $\cdots$ \\
\hline 103 & $\mathrm{~N}$ & $\ldots$ & $\ldots$ & $\ldots$ & $\cdots$ & $\ldots$ \\
\hline 115 & $\mathrm{Y}$ & $78 \pm 102$ & $\mathrm{~N}$ & $\ldots$ & $\ldots$ & $\ldots$ \\
\hline 134 & $\mathrm{~N}$ & $\ldots$ & $\cdots$ & $\cdots$ & $\cdots$ & $\cdots$ \\
\hline 151 & $\mathrm{~N}$ & $\ldots$ & $\mathrm{N}$ & $\ldots$ & $\cdots$ & $\ldots$ \\
\hline 154 & $\mathrm{Y}$ & $37 \pm 10$ & $\mathrm{Y}$ & $152 \pm 3$ & $\cdots$ & $\cdots$ \\
\hline 173 & $\mathrm{~N}$ & $\ldots$ & $\cdots$ & $\ldots$ & $\cdots$ & $\cdots$ \\
\hline 199 & $\mathrm{~N}$ & $\ldots$ & $\ldots$ & $\ldots$ & $\cdots$ & $\cdots$ \\
\hline 200 & $\mathrm{~N}$ & $\ldots$ & $\mathrm{Y}$ & $71 \pm 7$ & $\cdots$ & $\cdots$ \\
\hline 209 & $\ldots$ & $\ldots$ & $\mathrm{N}$ & $\ldots$ & $\ldots$ & $\ldots$ \\
\hline 213 & $\mathrm{Y}$ & $158 \pm 68$ & $\mathrm{Y}$ & $77 \pm 29$ & $\ldots$ & $\cdots$ \\
\hline 219 & $\mathrm{~N}$ & $\ldots$ & $\ldots$ & $\ldots$ & $\ldots$ & $\ldots$ \\
\hline 222 & $\mathrm{~N}$ & $\ldots$ & $\cdots$ & $\cdots$ & $\cdots$ & $\cdots$ \\
\hline 229 & $\mathrm{Y}$ & $29 \pm 574$ & $\mathrm{~N}$ & $\ldots$ & $\ldots$ & $\ldots$ \\
\hline 235 & $\mathrm{~N}$ & $\ldots$ & $\ldots$ & $\ldots$ & $\cdots$ & $\ldots$ \\
\hline 236 & $\mathrm{~N}$ & $\ldots$ & $\cdots$ & $\ldots$ & $\cdots$ & $\ldots$ \\
\hline 237 & $\mathrm{~N}$ & $\ldots$ & $\ldots$ & $\ldots$ & $\ldots$ & $\ldots$ \\
\hline 238 & $\mathrm{Y}$ & $64 \pm 9$ & $\ldots$ & $\ldots$ & $\ldots$ & $\ldots$ \\
\hline 243 & $\mathrm{~N}$ & $\ldots$ & $\ldots$ & $\ldots$ & $\cdots$ & $\cdots$ \\
\hline 246 & $\mathrm{Y}$ & $45 \pm 22$ & $\ldots$ & $\ldots$ & $\ldots$ & $\ldots$ \\
\hline 253 & $\mathrm{~N}$ & $\ldots$ & $\ldots$ & $\ldots$ & $\ldots$ & $\ldots$ \\
\hline 257 & $\mathrm{Y}$ & $122 \pm 72$ & $\ldots$ & $\ldots$ & $\ldots$ & $\ldots$ \\
\hline 259 & $\mathrm{Y}$ & $125 \pm 74$ & $\ldots$ & $\ldots$ & $\ldots$ & $\ldots$ \\
\hline 262 & $\mathrm{~N}$ & $\ldots$ & $\cdots$ & $\cdots$ & $\cdots$ & $\cdots$ \\
\hline 265 & $\mathrm{Y}$ & $129 \pm 21$ & $\ldots$ & $\ldots$ & $\ldots$ & $\ldots$ \\
\hline 270 & $\ldots$ & $\ldots$ & $\mathrm{N}$ & $\ldots$ & $\ldots$ & $\ldots$ \\
\hline 271 & $\mathrm{~N}$ & $\ldots$ & $\ldots$ & $\ldots$ & $\ldots$ & $\ldots$ \\
\hline 275 & $\cdots$ & $\ldots$ & $\cdots$ & $\ldots$ & $\mathrm{Y}$ & $100 \pm 4$ \\
\hline 278 & $\ldots$ & $\ldots$ & $\ldots$ & $\ldots$ & $\mathrm{N}$ & $\ldots$ \\
\hline 279 & $\mathrm{Y}$ & $20 \pm 26$ & $\mathrm{~N}$ & $\ldots$ & $\ldots$ & $\ldots$ \\
\hline 280 & $\mathrm{~N}$ & $\ldots$ & $\mathrm{N}$ & $\ldots$ & $\cdots$ & $\ldots$ \\
\hline 281 & $\ldots$ & $\ldots$ & $\mathrm{Y}$ & $28 \pm 12$ & $\mathrm{~N}$ & $\ldots$ \\
\hline 284 & $\mathrm{~N}$ & $\ldots$ & $\ldots$ & $\ldots$ & $\ldots$ & $\ldots$ \\
\hline 286 & $\mathrm{~N}$ & $\ldots$ & $\ldots$ & $\ldots$ & $\ldots$ & $\ldots$ \\
\hline 287 & $\mathrm{~N}$ & $\ldots$ & $\mathrm{N}$ & $\ldots$ & $\ldots$ & $\cdots$ \\
\hline 289 & $\mathrm{~N}$ & $\cdots$ & $\ldots$ & $\ldots$ & $\cdots$ & $\cdots$ \\
\hline 294 & $\mathrm{~N}$ & $\cdots$ & $\mathrm{Y}$ & $52 \pm 22$ & $\cdots$ & $\ldots$ \\
\hline 297 & $\mathrm{~N}$ & $\ldots$ & $\mathrm{Y}$ & $25 \pm 21$ & $\ldots$ & $\ldots$ \\
\hline 301 & $\mathrm{Y}$ & $29 \pm 27$ & $\ldots$ & $\ldots$ & $\ldots$ & $\ldots$ \\
\hline 307 & $\mathrm{Y}$ & $38 \pm 19$ & $\mathrm{Y}$ & $67 \pm 8$ & $\mathrm{~N}$ & $\ldots$ \\
\hline 308 & $\mathrm{Y}$ & $19 \pm 30$ & $\mathrm{Y}$ & $38 \pm 14$ & $\ldots$ & $\ldots$ \\
\hline 313 & $\mathrm{~N}$ & $\ldots$ & $\ldots$ & $\ldots$ & $\cdots$ & $\ldots$ \\
\hline 315 & $\mathrm{~N}$ & $\ldots$ & $\ldots$ & $\ldots$ & $\ldots$ & $\ldots$ \\
\hline 319 & $\mathrm{~N}$ & $\ldots$ & $\ldots$ & $\ldots$ & $\ldots$ & $\ldots$ \\
\hline 325 & $\mathrm{~N}$ & $\ldots$ & $\ldots$ & $\ldots$ & $\ldots$ & $\ldots$ \\
\hline 330 & $\mathrm{~N}$ & $\ldots$ & $\ldots$ & $\ldots$ & $\ldots$ & $\ldots$ \\
\hline 331 & $\ldots$ & $\ldots$ & $\mathrm{N}$ & $\ldots$ & $\ldots$ & $\ldots$ \\
\hline 332 & $\mathrm{~N}$ & $\cdots$ & $\ldots$ & $\cdots$ & $\cdots$ & $\cdots$ \\
\hline 333 & $\ldots$ & $\ldots$ & $\mathrm{N}$ & $\ldots$ & $\ldots$ & $\ldots$ \\
\hline 341 & $\mathrm{Y}$ & $51 \pm 10$ & $\mathrm{Y}$ & $55 \pm 13$ & $\mathrm{~N}$ & $\ldots$ \\
\hline 344 & $\ldots$ & $\ldots$ & $\mathrm{N}$ & $\ldots$ & $\ldots$ & $\ldots$ \\
\hline 357 & $\ldots$ & $\ldots$ & $\mathrm{N}$ & $\ldots$ & $\ldots$ & $\ldots$ \\
\hline 358 & $\cdots$ & $\cdots$ & $\mathrm{N}$ & $\ldots$ & $\cdots$ & $\cdots$ \\
\hline 364 & $\ldots$ & $\ldots$ & $\mathrm{Y}$ & $73 \pm 16$ & $\ldots$ & $\ldots$ \\
\hline 365 & $\ldots$ & $\ldots$ & $\mathrm{N}$ & $\ldots$ & $\ldots$ & $\ldots$ \\
\hline 371 & $\ldots$ & $\ldots$ & $\mathrm{N}$ & $\ldots$ & $\ldots$ & $\ldots$ \\
\hline 373 & $\ldots$ & $\ldots$ & $\ldots$ & $\ldots$ & $\mathrm{Y}$ & $91 \pm 7$ \\
\hline 374 & $\mathrm{~N}$ & $\ldots$ & $\mathrm{N}$ & $\ldots$ & $\ldots$ & $\ldots$ \\
\hline 380 & $\mathrm{~N}$ & $\ldots$ & $\ldots$ & $\ldots$ & $\ldots$ & $\ldots$ \\
\hline 382 & $\mathrm{~N}$ & $\cdots$ & $\ldots$ & $\ldots$ & $\cdots$ & $\cdots$ \\
\hline 383 & $\mathrm{~N}$ & $\ldots$ & $\mathrm{Y}$ & $39 \pm 17$ & $\ldots$ & $\ldots$ \\
\hline
\end{tabular}


Table 4

(Continued)

\begin{tabular}{|c|c|c|c|c|c|c|}
\hline ID & $1.3 \mathrm{~cm}$ Variable? & $(\Delta F / F)_{1.3 \mathrm{~cm}}$ & $3.6 \mathrm{~cm}$ Variable? & $(\Delta F / F)_{3.6 \mathrm{~cm}}$ & $6 \mathrm{~cm}$ Variable? & $(\Delta F / F)_{6 \mathrm{~cm}}$ \\
\hline 384 & $\cdots$ & $\ldots$ & $\mathrm{N}$ & $\ldots$ & $\ldots$ & $\ldots$ \\
\hline 386 & $\ldots$ & $\ldots$ & $\mathrm{Y}$ & $36 \pm 20$ & $\ldots$ & $\ldots$ \\
\hline 389 & $\mathrm{~N}$ & $\ldots$ & $\mathrm{Y}$ & $70 \pm 4$ & $\ldots$ & $\ldots$ \\
\hline 391 & $\ldots$ & $\ldots$ & $\mathrm{N}$ & $\ldots$ & $\mathrm{N}$ & $\ldots$ \\
\hline 393 & $\mathrm{~N}$ & $\ldots$ & $\ldots$ & $\ldots$ & $\ldots$ & $\ldots$ \\
\hline 394 & $\mathrm{Y}$ & $72 \pm 9$ & $\ldots$ & $\ldots$ & $\ldots$ & $\ldots$ \\
\hline 399 & $\cdots$ & $\ldots$ & $\mathrm{N}$ & $\ldots$ & $\cdots$ & $\cdots$ \\
\hline 400 & $\cdots$ & $\ldots$ & $\mathrm{Y}$ & $60 \pm 16$ & $\ldots$ & $\ldots$ \\
\hline 401 & $\mathrm{~N}$ & $\ldots$ & $\ldots$ & $\ldots$ & $\ldots$ & $\ldots$ \\
\hline 407 & $\cdots$ & $\ldots$ & $\ldots$ & $\ldots$ & $\mathrm{Y}$ & $101 \pm 3$ \\
\hline 408 & $\mathrm{~N}$ & $\ldots$ & $\mathrm{Y}$ & $31 \pm 21$ & $\mathrm{~N}$ & $\ldots$ \\
\hline 409 & $\mathrm{~N}$ & $\ldots$ & $\ldots$ & $\ldots$ & $\ldots$ & $\ldots$ \\
\hline 411 & $\cdots$ & $\ldots$ & $\ldots$ & $\ldots$ & $\mathrm{N}$ & $\ldots$ \\
\hline 413 & $\mathrm{Y}$ & $41 \pm 14$ & $\mathrm{Y}$ & $23 \pm 15$ & $\mathrm{~N}$ & $\ldots$ \\
\hline 414 & $\cdots$ & $\ldots$ & $\mathrm{N}$ & $\ldots$ & $\cdots$ & $\ldots$ \\
\hline 416 & $\ldots$ & $\ldots$ & $\mathrm{N}$ & $\ldots$ & $\mathrm{N}$ & $\ldots$ \\
\hline 418 & $\mathrm{Y}$ & $88 \pm 5$ & $\mathrm{Y}$ & $63 \pm 10$ & $\ldots$ & $\ldots$ \\
\hline 421 & $\mathrm{~N}$ & $\ldots$ & $\mathrm{N}$ & $\ldots$ & $\ldots$ & $\ldots$ \\
\hline 423 & $\cdots$ & $\ldots$ & $\mathrm{N}$ & $\ldots$ & $\mathrm{N}$ & $\ldots$ \\
\hline 427 & $\mathrm{~N}$ & $\ldots$ & $\mathrm{Y}$ & $33 \pm 13$ & $\ldots$ & $\ldots$ \\
\hline 428 & $\mathrm{~N}$ & $\ldots$ & $\ldots$ & $\ldots$ & $\ldots$ & $\ldots$ \\
\hline 430 & $\ldots$ & $\ldots$ & $\mathrm{N}$ & $\ldots$ & $\mathrm{N}$ & $\ldots$ \\
\hline 433 & $\ldots$ & $\ldots$ & $\mathrm{Y}$ & $39 \pm 13$ & $\ldots$ & $\ldots$ \\
\hline 437 & $\mathrm{Y}$ & $284 \pm 45$ & $\mathrm{Y}$ & $215 \pm 46$ & $\mathrm{~N}$ & $\cdots$ \\
\hline 438 & $\mathrm{~N}$ & $\ldots$ & $\mathrm{N}$ & $\ldots$ & $\mathrm{N}$ & $\ldots$ \\
\hline 439 & $\mathrm{Y}$ & $24 \pm 32$ & $\mathrm{~N}$ & $\ldots$ & $\mathrm{N}$ & $\ldots$ \\
\hline 440 & $\mathrm{Y}$ & $299 \pm 89$ & $\ldots$ & $\ldots$ & $\ldots$ & $\ldots$ \\
\hline 442 & $\ldots$ & $\ldots$ & $\mathrm{Y}$ & $29 \pm 17$ & $\ldots$ & $\ldots$ \\
\hline 444 & $\mathrm{~N}$ & $\ldots$ & $\mathrm{N}$ & $\ldots$ & $\ldots$ & $\ldots$ \\
\hline 460 & $\mathrm{~N}$ & $\ldots$ & $\mathrm{N}$ & $\ldots$ & $\mathrm{N}$ & $\ldots$ \\
\hline 465 & $\mathrm{~N}$ & $\cdots$ & $\mathrm{N}$ & $\ldots$ & $\cdots$ & $\cdots$ \\
\hline 466 & $\mathrm{~N}$ & $\ldots$ & $\mathrm{Y}$ & $51 \pm 13$ & $\ldots$ & $\ldots$ \\
\hline 469 & $\mathrm{Y}$ & $108 \pm 21$ & $\ldots$ & $\ldots$ & $\ldots$ & $\ldots$ \\
\hline 473 & $\mathrm{~N}$ & $\ldots$ & $\mathrm{Y}$ & $55 \pm 11$ & $\ldots$ & $\ldots$ \\
\hline 477 & $\ldots$ & $\ldots$ & $\mathrm{Y}$ & $60 \pm 16$ & $\ldots$ & $\ldots$ \\
\hline 481 & $\mathrm{~N}$ & $\ldots$ & $\ldots$ & $\ldots$ & $\ldots$ & $\ldots$ \\
\hline 483 & $\mathrm{~N}$ & $\ldots$ & $\mathrm{N}$ & $\ldots$ & $\ldots$ & $\ldots$ \\
\hline 484 & $\mathrm{~N}$ & $\ldots$ & $\mathrm{N}$ & $\ldots$ & $\ldots$ & $\ldots$ \\
\hline 491 & $\mathrm{~N}$ & $\ldots$ & $\mathrm{N}$ & $\ldots$ & $\mathrm{N}$ & $\ldots$ \\
\hline 492 & $\mathrm{Y}$ & $57 \pm 13$ & $\ldots$ & $\ldots$ & $\ldots$ & $\ldots$ \\
\hline 493 & $\ldots$ & $\ldots$ & $\ldots$ & $\ldots$ & $\mathrm{Y}$ & $102 \pm 3$ \\
\hline 494 & $\mathrm{~N}$ & $\ldots$ & $\mathrm{N}$ & $\ldots$ & $\ldots$ & $\ldots$ \\
\hline 499 & $\mathrm{Y}$ & $28 \pm 22$ & $\mathrm{~N}$ & $\ldots$ & $\ldots$ & $\ldots$ \\
\hline 501 & $\mathrm{~N}$ & $\ldots$ & $\mathrm{N}$ & $\ldots$ & $\cdots$ & $\ldots$ \\
\hline 512 & $\ldots$ & $\ldots$ & $\mathrm{Y}$ & $27 \pm 22$ & $\mathrm{~N}$ & $\ldots$ \\
\hline 516 & $\ldots$ & $\ldots$ & $\mathrm{N}$ & $\ldots$ & $\mathrm{N}$ & $\ldots$ \\
\hline 518 & $\mathrm{~N}$ & $\ldots$ & $\mathrm{N}$ & $\ldots$ & $\mathrm{N}$ & $\ldots$ \\
\hline 519 & $\ldots$ & $\ldots$ & $\mathrm{N}$ & $\ldots$ & $\ldots$ & $\ldots$ \\
\hline 529 & $\mathrm{Y}$ & $99 \pm 11$ & $\ldots$ & $\ldots$ & $\ldots$ & $\ldots$ \\
\hline 530 & $\ldots$ & $\ldots$ & $\mathrm{N}$ & $\ldots$ & $\ldots$ & $\ldots$ \\
\hline 535 & $\mathrm{~N}$ & $\ldots$ & $\mathrm{Y}$ & $31 \pm 19$ & $\ldots$ & $\ldots$ \\
\hline 537 & $\ldots$ & $\ldots$ & $\mathrm{N}$ & $\ldots$ & $\ldots$ & $\ldots$ \\
\hline 544 & $\mathrm{Y}$ & $102 \pm 4$ & $\ldots$ & $\ldots$ & $\ldots$ & $\ldots$ \\
\hline 546 & $\mathrm{~N}$ & $\ldots$ & $\mathrm{N}$ & $\ldots$ & $\ldots$ & $\ldots$ \\
\hline 549 & $\mathrm{~N}$ & $\ldots$ & $\ldots$ & $\ldots$ & $\ldots$ & $\ldots$ \\
\hline 555 & $\mathrm{~N}$ & $\ldots$ & $\mathrm{N}$ & $\ldots$ & $\mathrm{N}$ & $\ldots$ \\
\hline 564 & $\ldots$ & $\ldots$ & $\mathrm{N}$ & $\ldots$ & $\mathrm{N}$ & $\ldots$ \\
\hline 587 & $\mathrm{Y}$ & $136 \pm 3$ & $\mathrm{Y}$ & $69 \pm 6$ & $\ldots$ & $\ldots$ \\
\hline 595 & $\ldots$ & $\ldots$ & $\mathrm{N}$ & $\ldots$ & $\mathrm{N}$ & $\ldots$ \\
\hline 599 & $\mathrm{~N}$ & $\ldots$ & $\ldots$ & $\cdots$ & $\ldots$ & $\cdots$ \\
\hline 605 & $\mathrm{~N}$ & $\ldots$ & $\ldots$ & $\ldots$ & $\ldots$ & $\ldots$ \\
\hline 608 & $\mathrm{~N}$ & $\ldots$ & $\mathrm{Y}$ & $38 \pm 18$ & $\cdots$ & $\ldots$ \\
\hline 616 & $\mathrm{~N}$ & $\ldots$ & $\ldots$ & $\ldots$ & $\ldots$ & $\ldots$ \\
\hline 617 & $\mathrm{Y}$ & $24 \pm 14$ & $\mathrm{Y}$ & $49 \pm 12$ & $\ldots$ & $\ldots$ \\
\hline
\end{tabular}


Table 4

(Continued)

\begin{tabular}{|c|c|c|c|c|c|c|}
\hline ID & $1.3 \mathrm{~cm}$ Variable? & $(\Delta F / F)_{1.3 \mathrm{~cm}}$ & $3.6 \mathrm{~cm}$ Variable? & $(\Delta F / F)_{3.6 \mathrm{~cm}}$ & $6 \mathrm{~cm}$ Variable? & $(\Delta F / F)_{6 \mathrm{~cm}}$ \\
\hline 621 & $\mathrm{~N}$ & $\ldots$ & $\ldots$ & $\ldots$ & $\ldots$ & $\ldots$ \\
\hline 649 & $\mathrm{~N}$ & $\ldots$ & $\ldots$ & $\ldots$ & $\ldots$ & $\ldots$ \\
\hline 658 & $\mathrm{~N}$ & $\ldots$ & $\ldots$ & $\ldots$ & $\ldots$ & $\ldots$ \\
\hline 665 & $\mathrm{~N}$ & $\ldots$ & $\ldots$ & $\ldots$ & $\ldots$ & $\ldots$ \\
\hline 668 & $\mathrm{~N}$ & $\ldots$ & $\ldots$ & $\ldots$ & $\ldots$ & $\ldots$ \\
\hline 690 & $\mathrm{~N}$ & $\ldots$ & $\ldots$ & $\ldots$ & $\ldots$ & $\ldots$ \\
\hline 715 & $\mathrm{Y}$ & $92 \pm 24$ & $\ldots$ & $\ldots$ & $\ldots$ & $\ldots$ \\
\hline 730 & $\mathrm{Y}$ & $51 \pm 9$ & $\mathrm{Y}$ & $50 \pm 6$ & $\mathrm{Y}$ & $47 \pm 11$ \\
\hline 737 & $\mathrm{Y}$ & $905 \pm 487$ & $\ldots$ & $\ldots$ & $\ldots$ & $\ldots$ \\
\hline 760 & $\mathrm{~N}$ & $\ldots$ & $\ldots$ & $\ldots$ & $\ldots$ & $\ldots$ \\
\hline 786 & $\mathrm{~N}$ & $\ldots$ & $\ldots$ & $\cdots$ & $\ldots$ & $\ldots$ \\
\hline 821 & $\mathrm{~N}$ & $\ldots$ & $\ldots$ & $\ldots$ & $\ldots$ & $\ldots$ \\
\hline 835 & $\mathrm{~N}$ & $\ldots$ & $\ldots$ & $\ldots$ & $\ldots$ & $\ldots$ \\
\hline 842 & $\mathrm{~N}$ & $\ldots$ & $\ldots$ & $\ldots$ & $\ldots$ & $\ldots$ \\
\hline 848 & $\mathrm{~N}$ & $\ldots$ & $\ldots$ & $\ldots$ & $\ldots$ & $\ldots$ \\
\hline 859 & $\mathrm{~N}$ & $\ldots$ & $\ldots$ & $\ldots$ & $\ldots$ & $\ldots$ \\
\hline 862 & $\mathrm{~N}$ & $\ldots$ & $\ldots$ & $\ldots$ & $\ldots$ & $\ldots$ \\
\hline 891 & $\mathrm{~N}$ & $\ldots$ & $\ldots$ & $\ldots$ & $\ldots$ & $\ldots$ \\
\hline 897 & $\mathrm{~N}$ & $\ldots$ & $\ldots$ & $\ldots$ & $\ldots$ & $\ldots$ \\
\hline
\end{tabular}

Below, we split the sources whose SEDs are not fitted well by our model and, therefore, are not indicative of being freefree emission, or do not follow the expected trend of decreasing centimeter flux with increasing separation from $\theta^{1}$ Ori $\mathrm{C}$ :

\subsubsection{Strong Free-Free Sources}

Source 418 is $\theta^{1}$ Ori A, a binary system with a B 0.5 primary star and a low-mass companion, possibly a T Tauri star (Levato \& Abt 1976; Bossi et al. 1989), which is known to be highly variable (e.g., Felli et al. 1993b). Rivilla et al. (2015) suggest that this variability may be twofold, (i) variations in free-free opacity from a stellar wind from the interactions with the companion, and (ii) variations in the non-thermal emission from stellar activity related to the distance between to binary, similar to the case of WR 140 (e.g., Williams et al. 1990). Rivilla et al. (2015) suggest that while the former mechanism may be present, the latter is required to explain previous observations.

Sources 279 and 308 each have radio spectra that are steeper than $\nu^{0.6}$. Source 279 is the Becklin-Neugebauer Object, and is thought to be a runaway B star, ejected from a system with Source I (our Source 308) in an explosive event 500 years ago (e.g., Plambeck et al. 1995; Gómez et al. 2008; Plambeck et al. 2013). The BN Object has a spectral dependence of $\nu^{1.3}$ below $100 \mathrm{GHz}$, above which it flattens, and is suggested to be freefree emission from a dense, hypercompact H II region. Source I has a spectral dependence of $\nu^{2}$ and is most easily explained by $\mathrm{H}^{-}$free-free emission in a disk (e.g., Plambeck et al. 2013). Both sources have massive stars driving ionizing circumstellar material and driving the free-free emission we detect, explaining their significant fluxes despite their distance from $\theta^{1}$ Ori C.

\subsubsection{Dust-only Sources}

Sources $134,236,246$, and 301 have millimeter $(850 \mu \mathrm{m}$ or $1.3 \mathrm{~mm}$ ) detections and are detected at $1.3 \mathrm{~cm}$ in our maps, but are undetected at 3.6 and $6 \mathrm{~cm}$. The SEDs for all of these sources are well fitted by a model that is predominantly dust emission at $1.3 \mathrm{~cm}$ (and perhaps a minor contribution from free-free emission). Sources 236 and 246 are identified by Eisner et al. (2008) as "MM" objects (MM21 and MM8 respectively), which lack near-IR counterparts. Source 301 is also identified by Eisner et al. (2008) as LMLA 162. All three of those sources $(236,246$, and 301) are among the most massive known sources in the ONC $\left(>0.2 \quad M_{\odot}\right.$ Eisner et al. 2008). They are likely highly embedded young objects, and may be candidate Class 0 or I objects as suggested by Eisner et al. (2008).

\subsubsection{Non-thermal Radio Sources}

Sources 11 and 617 each have spectra with steep negative spectral indices (see Figure 5), which may indicate that they are emitting synchrotron radiation. Source 11 is not associated with any previous detections in our reference catalogs, and is not found to not be variable at $3.6 \mathrm{~cm}$. Source 617 has previously been detected, and is commonly referred to as F (e.g., Churchwell et al. 1987; Garay et al. 1987; Felli et al. 1993a). It has previously been found to experience radio flares on timescales as short as hours and possibly as long as months (Rivilla et al. 2015).

Sources 154, 394, 437, 440, 529, 544, 587, and 730 are highly variable sources, showing significant changes in flux over just a few days between our observations on 2014 March 3 and 7. All are significant outliers in Figure 5. Source 154 has previously been identified as A (e.g., Churchwell et al. 1987; Garay et al. 1987; Felli et al. 1993a), and Zapata et al. (2004a) find the source to show large percentages of circular polarization, and suggest that the emission may be gyrosynchrotron in nature. Felli et al. (1993b) also classify Source 587, also known as $\mathrm{G}$, as a non-thermal variable emitter. All of these sources are associated with infrared detected objects (Hillenbrand \& Carpenter 2000). These sources may be indicative of radio flares of gyrosynchrotron emission, but further observations with concurrent flux measurements at multiple wavelengths are needed to confirm this.

Source 903 is located far from $\theta^{1}$ Ori C, and is only detected at $6 \mathrm{~cm}$, but it has a high flux given its significant separation (see Figure 5). It is out of the field of view of our $1.3 \mathrm{~cm}$ data, 
and right on the edge of our $3.6 \mathrm{~cm}$ map, but undetected. It is associated with the proplyd 281-306, which is a disk seen only in silhouette with HST (Ricci et al. 2008). Radio emission from this source may be attributed to magnetic activity from the young star or free-free emission from material ionized by the star itself, as it is likely too far to be material ionized by $\theta^{1}$ Ori C.

Source 904 is also located far from $\theta^{1}$ Ori C, with a high flux given its separation (Figure 5), and is outside the field of view of our $1.3 \mathrm{~cm}$ map. Its 6 and $3.6 \mathrm{~cm}$ fluxes are consistent with free-free emission, but do show indications that the spectral index may be significantly negative. It is unassociated with any previous catalog.

\subsubsection{Extragalactic Sources}

Given the large survey area of our maps, it is possible that some of our detections are extragalactic in nature. Following Fomalont et al. (1991), at $6 \mathrm{~cm}$ we would expect the number of extragalactic contaminants greater than $156 \mu \mathrm{Jy}$ (our $6 \sigma$ threshold) in our $109 \mathrm{arcmin}^{2}$ survey area to be $6.5 \pm 2.3$. At $3.6 \mathrm{~cm}$, using Fomalont et al. (2002), we estimate $1.1 \pm 0.2$ extragalactic sources in our $70 \mathrm{arcmin}^{2}$ survey area to above $216 \mu \mathrm{Jy}$. No similar survey exists at $1.3 \mathrm{~cm}$, and so we use the $3.6 \mathrm{~cm}$ numbers to estimate that in our $1.3 \mathrm{~cm}$ map we would expect $1.8 \pm 0.2$ contaminants in our $34 \operatorname{arcmin}^{2}$ survey area above $72 \mu \mathrm{Jy}$. As most of these sources show non-thermal emission with negative spectral indices at these wavelengths (Condon 1992) they should be fainter at $1.3 \mathrm{~cm}$ than at $3.6 \mathrm{~cm}$ and therefore we would expect the contamination at $1.3 \mathrm{~cm}$ to be even smaller than this.

\subsection{Free-Free Contamination of Submillimeter Dust Masses}

At submillimeter, millimeter and radio wavelengths, the light emitted by dust grains is expected to be largely optically thin and the flux is directly proportional to the amount of dust present (e.g., Beckwith et al. 1990). As such, submillimeter flux measurements of protoplanetary disks are commonly used to measure the mass of those disks (e.g., Andrews \& Williams 2005; Eisner et al. 2008; Mann \& Williams 2010; Andrews et al. 2013; Mann et al. 2014). A number of previous surveys across millimeter and submillimeter wavelengths have employed this method to measure disk masses for protoplanetary disks in the ONC (e.g., Mundy et al. 1995; Bally et al. 1998b; Williams et al. 2005; Eisner \& Carpenter 2006; Eisner et al. 2008; Mann \& Williams 2010; Mann et al. 2014).

The proplyds, however, are located near the Trapezium Cluster of young massive stars that are photoevaporating the disks (e.g., Churchwell et al. 1987). The ionized material produced in the proplyds emits free-free emission, which can be bright at the same wavelengths used to measure disk masses. In order to accurately measure disk masses, it is, therefore, important to separate the dust and free-free contributions to submillimeter and millimeter fluxes. This is particularly true with the advent of ALMA, which will detect disks in the ONC much fainter than those that have been previously detected.

In this work, we characterized the free-free emission from a collection of compact radio sources in the ONC. In Table 3 we use the best fit free-free emission spectrum model from Section 3.2 to calculate the expected free-free flux at all ALMA bands. This table can be used to correct measured millimeter fluxes for free-free contamination, and accurately measure the submillimeter dust flux and thereby the dust mass.

For most sources, this extrapolation provides a good estimate of the free-free contribution of the source at ALMA wavelengths. This is not true, however, of sources for which the model fit is poor, as was discussed in the previous section. Furthermore, the extrapolation to ALMA bands for sources whose turnover frequency is designated as $>22 \mathrm{GHz}$ is also very uncertain. Many of these sources were only detected at $1.3 \mathrm{~cm}$, and a few have radio photometry that is best fitted by a $\nu^{0.6}$ power law. Our extrapolation for these sources assumes $\nu_{\text {turn }}=22 \mathrm{GHz}$, but if $\nu_{\text {turn }}>22 \mathrm{GHz}$ the free-free flux at ALMA bands would be greater than our current prediction. Further radio or millimeter observations are necessary to constrain $\nu_{\text {turn }}$ before accurate ALMA free-free fluxes can be predicted.

Variability is also a significant source of uncertainty in determining how well free-free emission from disks can be constrained and removed from disk mass measurements, if the free-free flux to dust flux ratio is large. For example, the measured $230 \mathrm{GHz}$ flux of Source 439 is $8.8 \mathrm{mJy}$ and the model free-free flux at $230 \mathrm{GHz}$ is $2.8 \mathrm{mJy}$, with a variability of $24 \%$. So free-free emission makes up $32 \pm 8 \%$ of the total $230 \mathrm{GHz}$ flux. Source 466, however, has a measured $230 \mathrm{GHz}$ flux of $7.7 \mathrm{mJy}, 0.3 \mathrm{mJy}$ of which is due to free-free emission with $50.8 \%$ variability, so the free-free emission makes up $5 \pm 2 \%$ of that flux. Because of the smaller free-free flux to total flux ratio of Source 466, the dust flux can be better constrained, even though Source 466 is more variable.

ALMA, however, will be able to detect disks which are much fainter than has previously been possible, For these sources, variability may be a significant problem. Source 469 has a $230 \mathrm{GHz}$ free-free flux of $0.33 \mathrm{mJy}$ with a variability of $108 \%$. Although it has no previous millimeter detections, if it were found to have a millimeter flux of $0.5 \mathrm{mJy}$, the dust mass calculation would be highly uncertain, because the free-free flux would make up $65 \pm 70 \%$ of the total $230 \mathrm{GHz}$ flux.

An accurate estimate of the uncertainty associated with variability of our sources, however, likely requires further monitoring of the sources to characterize the timescale and amplitude of the variability. Sources with significant variability may even require concurrent millimeter and radio flux measurements in order to measure the free-free contribution to millimeter flux measurements.

While not important for some objects, the correction for free-free emission is often crucial for correctly measuring disk mass. For example, the free-free emission from sources 391, $408,416,418,421,423,430,438,439,442,446,460,465$, 484, 491, 494, 499, 512, 516, 518, 535, 537, 555, 564, 605, 612 , and 617 contributes $>50 \%$ of the measured $3 \mathrm{~mm}$ fluxes. Free-free emission also contributes $>40 \%$ of the measured $1.3 \mathrm{~mm}$ fluxes for sources 408, 416, 418, 421, 423, 438, 442, $460,465,484,491,494,499,516,518,535,564$, and 617 and $>30 \%$ of the measured $850 \mu \mathrm{m}$ fluxes for sources 408,421 , $423,438,460,465,484,491,494,499$, and 617. Without these corrections, disk mass estimates from these submillimeter bands would be off by a significant amount.

\subsection{Future Work}

While our radio data set is tremendously useful for finding radio sources and characterizing their free-free emission for ALMA disk mass studies in the ONC, the data also have a 
number of other applications which we will explore in future work.

Due to the high resolution of our maps, particularly at $1.3 \mathrm{~cm}$, many of the sources detected in our maps are well resolved. The morphology of these objects show interesting features, particularly when matched up with high-resolution $H S T$ images of the proplyds. For many sources, structure in the HST maps is well matched with features in our maps.

Furthermore, we can use resolved images to measure massloss rates for the protoplanetary disks. The free-free emission we detect here originates from ionized cocoons of gas, which are flowing away from their associated disks under the intense radiation pressure from the star $\theta^{1}$ Ori $\mathrm{C}$. The flux of this freefree emission coupled with measured sizes of these cocoons of gas is sufficient to measure disk mass-loss rates. Mass-loss rates have previously been measured for a handful of disks in the ONC (e.g., Churchwell et al. 1987), but the improved sensitivity and resolution of our maps will allow us to make this measurement for many more sources.

\section{CONCLUSIONS}

We have produced new high spatial resolution maps of the Orion Nebula at 1.3,3.6, and $6 \mathrm{~cm}$ with significantly improved sensitivities compared with previous radio studies of the region using the JVLA. In these maps, we search for compact $\left(\lesssim 2^{\prime \prime}\right)$ radio sources, and use these detections to constrain the properties of free-free emission from protoplanetary disks in the ONC. Free-free emission is emitted from the ionized winds driven by the nearby massive star $\theta^{1}$ Ori C. Constraints on this free-free emission are crucial for studies aiming to measure disk masses for the proplyds from submillimeter fluxes.

We detect 144 sources at $1.3 \mathrm{~cm}, 98$ sources at $3.6 \mathrm{~cm}$, and 108 sources at $6 \mathrm{~cm}$, for a total of 175 unique sources. Of these 175 detections, 149 have previously been detected at radio wavelengths, 67 are associated with HST-detected proplyds, 120 with near-infrared-detected YSOs, 40 with sources detected previously at millimeter wavelengths, and 11 are detected for the first time at any wavelength.

For each source detected in our maps we report its position and flux, as measured by fitting a Gaussian to the source, in Table 2. For previously identified sources not detected in one or more of our maps, we also report the integrated flux in a $1^{\prime \prime}$ aperture measured toward the source. This information is presented in an extended version of Table 2 that is available in the online material.

We fit each of our source spectra with a combined dust + free-free emission model. The majority of our targets are fitted well by this dust + free-free model, with many showing evidence of a turnover in the free-free emission. Further studies of free-free emission may benefit from longer wavelength flux measurements to better constrain the free-free turnover. Four of our detected sources (134, 236, 246, and 301) have SEDs that are consistent with being produced entirely by dust emission and are likely highly embedded young objects. We also detect the Becklin-Neugebauer Object, its alleged counterpart Source I, and $\theta^{1}$ Ori A.

Many of our sources have previously measured radio fluxes, and so we can investigate variability. We find that 30 sources are variable at $1.3 \mathrm{~cm}, 32$ at $3.6 \mathrm{~cm}$, and 5 at $6 \mathrm{~cm}$. 55 of our detected sources are variable at one or more wavelengths. For sources that are variable we define a metric, $\Delta F / F$, to quantify the variability, and find that $\Delta F / F \approx 20 \%-900 \%$ for our targets. 13 are variable at $>100 \%$, suggesting that any submillimeter measurements will be very uncertain. The time sampling is, however, poor, and so more dedicated monitoring of our targets is necessary for better understanding this variability.

Finally, the free-free emission properties derived from our modeling can be extrapolated to submillimeter wavelengths to estimate the free-free contribution to submillimeter fluxes. This is necessary for correctly distinguishing dust emission and free-free emission at submillimeter wavelengths, particularly when submillimeter fluxes are used to calculate disk dust masses. This will be crucial for future studies of dust emission from protoplanetary disks in the ONC with ALMA. We provide free-free flux estimates for each detected source at each ALMA band in Table 3. Variability is a significant source of uncertainty in correcting millimeter flux measurements for free-free emission if the free-free flux to dust flux ratio is large, and so understanding this variability is an important future direction.

In the future, we will use this data set to study the morphology of the sources resolved in our high-resolution VLA maps, particularly compared with HST images of the proplyds. We will also measure the rate at which material is being photoevaporated and lost from the disks of these sources under the intense radiation and winds from $\theta^{1}$ Ori $\mathrm{C}$ and the Trapezium Cluster, and, therefore, derive disk lifetimes for the protoplanetary disks in the ONC.

This material is based upon work supported by the National Science Foundation Graduate Research Fellowship under Grant No. 2012115762. This work was supported by NSF AAG Grant 1311910. The results reported herein benefited from collaborations and/or information exchange within NASA's Nexus for Exoplanet System Science (NExSS) research coordination network sponsored by NASA's Science Mission Directorate. The National Radio Astronomy Observatory is a facility of the National Science Foundation operated under cooperative agreement by Associated Universities, Inc.

\section{REFERENCES}

Andrews, S. M., Rosenfeld, K. A., Kraus, A. L., \& Wilner, D. J. 2013, ApJ, 771,129

Andrews, S. M., \& Williams, J. P. 2005, ApJ, 631, 1134

Bally, J., Sutherland, R. S., Devine, D., \& Johnstone, D. 1998a, AJ, 116, 293

Bally, J., Testi, L., Sargent, A., \& Carlstrom, J. 1998b, AJ, 116, 854

Beckwith, S. V. W., Sargent, A. I., Chini, R. S., \& Guesten, R. 1990, AJ, 99, 924

Bossi, M., Gaspani, A., Scardia, M., \& Tadini, M. 1989, A\&A, 222, 117

Bower, G. C., Plambeck, R. L., Bolatto, A., et al. 2003, ApJ, 598, 1140

Caillault, J.-P., Gagné, M., \& Stauffer, J. R. 1994, ApJ, 432, 386

Churchwell, E., Felli, M., Wood, D. O. S., \& Massi, M. 1987, ApJ, 321, 516

Clark, B. G. 1980, A\&A, 89, 377

Condon, J. J. 1992, ARA\&A, 30, 575

Cornwell, T. J. 1988, A\&A, 202, 316

Dulk, G. A. 1985, ARA\&A, 23, 169

Dzib, S. A., Loinard, L., Mioduszewski, A. J., et al. 2013, ApJ, 775, 63

Eisner, J. A., \& Carpenter, J. M. 2006, ApJ, 641, 1162

Eisner, J. A., Hillenbrand, L. A., White, R. J., et al. 2007, ApJ, 669, 1072

Eisner, J. A., Plambeck, R. L., Carpenter, J. M., et al. 2008, ApJ, 683, 304

Feigelson, E. D., \& Montmerle, T. 1999, ARA\&A, 37, 363

Felli, M., Churchwell, E., Wilson, T. L., \& Taylor, G. B. 1993a, A\&AS, 98, 137

Felli, M., Taylor, G. B., Catarzi, M., Churchwell, E., \& Kurtz, S. 1993b, A\&AS, 101, 127

Fomalont, E. B., Kellermann, K. I., Partridge, R. B., Windhorst, R. A., \& Richards, E. A. 2002, AJ, 123, 2402 
Fomalont, E. B., Windhorst, R. A., Kristian, J. A., \& Kellerman, K. I. 1991, AJ, 102, 1258

Forbrich, J., Menten, K. M., \& Reid, M. J. 2008, A\&A, 477, 267

Forbrich, J., Preibisch, T., \& Menten, K. M. 2006, A\&A, 446, 155

Forbrich, J., Rivilla, V. M., Menten, K. M., et al. 2016, arXiv:1603.05666

Fullerton, A. W. 2003, in ASP Conf. Ser. 305, Magnetic Fields in O, B and

A Stars: Origin and Connection to Pulsation, Rotation and Mass Loss, ed.

L. A. Balona, H. F. Henrichs, \& R. Medupe (San Francisco, CA: ASP), 333

Garay, G., Moran, J. M., \& Reid, M. J. 1987, ApJ, 314, 535

Gómez, L., Rodríguez, L. F., Loinard, L., et al. 2008, ApJ, 685, 333

Gorti, U., \& Hollenbach, D. 2009, ApJ, 690, 1539

Henney, W. J., \& Arthur, S. J. 1998, AJ, 116, 322

Hillenbrand, L. A., \& Carpenter, J. M. 2000, ApJ, 540, 236

Hollenbach, D., Johnstone, D., Lizano, S., \& Shu, F. 1994, ApJ, 428, 654

Kenyon, S. J. 2001, in ASP Conf. Ser. 231, Tetons 4: Galactic Structure, Stars

and the Interstellar Medium, ed. C. E. Woodward, M. D. Bicay, \& J. M. Shull (San Francisco, CA: ASP), 594

Kounkel, M., Hartmann, L., Loinard, L., et al. 2014, ApJ, 790, 49

Levato, H., \& Abt, H. A. 1976, PASP, 88, 712

Mann, R. K., Di Francesco, J., Johnstone, D., et al. 2014, ApJ, 784, 82

Mann, R. K., \& Williams, J. P. 2009, ApJL, 694, L36

Mann, R. K., \& Williams, J. P. 2010, ApJ, 725, 430

Moran, J. M., Garay, G., Reid, M. J., Genzel, R., \& Ho, P. T. P. 1982, NYASA, 395, 204

Mundy, L. G., Looney, L. W., \& Lada, E. A. 1995, ApJL, 452, L137

O’Dell, C. R., \& Wen, Z. 1994, ApJ, 436, 194

O’Dell, C. R., Wen, Z., \& Hu, X. 1993, ApJ, 410, 696
Perley, R., Napier, P., Jackson, J., et al. 2009, IEEEP, 97, 1448

Plambeck, R. L., Bolatto, A. D., Carpenter, J. M., et al. 2013, ApJ, 765, 40

Plambeck, R. L., Wright, M. C. H., Mundy, L. G., \& Looney, L. W. 1995, ApJL, 455, L189

Rau, U., \& Cornwell, T. J. 2011, A\&A, 532, 71

Ricci, L., Robberto, M., \& Soderblom, D. R. 2008, AJ, 136, 2136

Ricci, L., Testi, L., Natta, A., et al. 2010a, A\&A, 512, A15

Ricci, L., Testi, L., Natta, A., \& Brooks, K. J. 2010b, A\&A, 521, A66

Rivilla, V. M., Chandler, C. J., Sanz-Forcada, J., et al. 2015, ApJ, 808, 146

Rodmann, J., Henning, T., Chandler, C. J., Mundy, L. G., \& Wilner, D. J. 2006, A\&A, 446, 211

Rodríguez-Ledesma, M. V., Mundt, R., \& Eislöffel, J. 2009, A\&A, 502, 883

Shu, F. H., Shang, H., Glassgold, A. E., \& Lee, T. 1997, Sci, 277, 1475

Smith, N., Bally, J., Shuping, R. Y., Morris, M., \& Kassis, M. 2005, AJ, 130, 1763

Stahl, O., Kaufer, A., Rivinius, T., et al. 1996, A\&A, 312, 539

Stahl, O., Wolf, B., Gang, T., et al. 1993, A\&A, 274, L29

Walborn, N. R., \& Nichols, J. S. 1994, ApJL, 425, L29

Williams, J. P., Andrews, S. M., \& Wilner, D. J. 2005, ApJ, 634, 495

Williams, P. M., van der Hucht, K. A., Pollock, A. M. T., et al. 1990, MNRAS, 243, 662

Wright, A. E., \& Barlow, M. J. 1975, MNRAS, 170, 41

Zapata, L. A., Rodríguez, L. F., Kurtz, S. E., \& O’Dell, C. R. 2004a, AJ, 127, 2252

Zapata, L. A., Rodríguez, L. F., Kurtz, S. E., O’Dell, C. R., \& Ho, P. T. P 2004b, ApJL, 610, L121 